\title{
Vector Addition Systems Reachability Problem (A Simpler Solution)
}

\author{
Jérôme Leroux* \\ LaBRI, Université de Bordeaux, CNRS
}

\begin{abstract}
The reachability problem for Vector Addition Systems (VASs) is a central problem of net theory. The general problem is known to be decidable by algorithms based on the classical Kosaraju-Lambert-Mayr-Sacerdote-Tenney decomposition (KLMST decomposition). Recently from this decomposition, we deduced that a final configuration is not reachable from an initial one if and only if there exists a Presburger inductive invariant that contains the initial configuration but not the final one. Since we can decide if a Preburger formula denotes an inductive invariant, we deduce from this result that there exist checkable certificates of non-reachability in the Presburger arithmetic. In particular, there exists a simple algorithm for deciding the general VAS reachability problem based on two semi-algorithms. A first one that tries to prove the reachability by enumerating finite sequences of actions and a second one that tries to prove the non-reachability by enumerating Presburger formulas. In another recent paper we provided the first proof of the VAS reachability problem that is not based on the KLMST decomposition. The proof is based on the notion of production relations that directly proves the existence of Presburger inductive invariants. In this paper we propose new intermediate results that dramatically simplify this last proof.
\end{abstract}

\section{Introduction}

Vector Addition Systems (VASs) or equivalently Petri Nets are one of the most popular formal methods for the representation and the analysis of parallel processes [2]. Their reachability problem is central since many computational problems (even outside the realm of parallel processes) reduce to the reachability problem. Sacerdote and Tenney provided in [13] a partial proof of decidability of this problem. The proof was completed in 1981 by Mayr [11] and simplified by Kosaraju [7] from [13, 11. Ten years later [8, Lambert provided a further simplified version based on [7. This last proof still remains difficult and the upper-bound complexity of the corresponding algorithm is just known to be non-primitive recursive. Nowadays, the exact complexity of the reachability problem for VASs is still an open-question. Even the existence of an elementary upper-bound complexity is open. In fact, the known general reachability algorithms are exclusively based on the Kosaraju-Lambert-Mayr-Sacerdote-Tenney (KLMST) decomposition.

Recently [9] we proved thanks to the KLMST decomposition that Parikh images of languages accepted by VASs are semi-pseudo-linear, a class that extends the Presburger sets. An application of this result was provided; we proved that a final configuration is not reachable from an initial one if and only if there exists a forward inductive invariant definable in the Presburger arithmetic that contains the initial configuration but not the final one. Since we can decide if a Presburger formula denotes a forward inductive invariant, we deduce that there exist checkable certificates of non-reachability in the Presburger arithmetic. In particular, there

*Work funded by ANR grant REACHARD-ANR-11-BS02-001. 
exists a simple algorithm for deciding the general VAS reachability problem based on two semialgorithms. A first one that tries to prove the reachability by enumerating finite sequences of actions and a second one that tries to prove the non-reachability by enumerating Presburger formulas.

In [10] we provided a new proof of the decidability of the reachability problem that does not introduce the KLMST decomposition. The proof is based on transformer relations and it proves that reachability sets are almost semilinear, a class of sets inspired by the class of semilinear sets [3] that extend the class of Presburger sets. Since the class of almost semilinear sets is strictly included in the class of semi-pseudo linear sets, this result is more precise than the one presented in [9]. This proof is based on a characterization of the conic sets definable in $\mathrm{FO}(\mathbb{Q},+, \leq)$ thanks to topological closures with vectors spaces. Unfortunately even though this characterization is simple, its proof is rather complex. In this paper we provide a more succinct and direct proof that transformer relations are definable in $\mathrm{FO}(\mathbb{Q},+, \leq)$. As a direct consequence topological properties on conic sets are no longer used in this new version.

Outline of the paper: Section 2 recalls the definition of almost semilinear sets, a class of sets inspired by the decomposition of Presburger sets into semilinear sets. Section 3 introduces definitions related to vector addition systems. Section 4 introduces a well-order over the runs of vector addition systems. This well-order is central in the proof and it was first introduced by Petr Jančar in another context [5. Based on the definition of this well-order we introduce in Section 5 the notion of transformer relations and we prove that conic relations generated by transformer relations are definable in $\mathrm{FO}(\mathbb{Q},+, \leq)$. Thanks to this result and the well-order introduced in the previous section we show in Section 6 that reachability sets of vector addition systems are almost semilinear. In Section 7 we introduce a dimension function for subsets of integer vectors. In Section 8 the almost semilinear sets are proved to be approximable by Presburger sets in a precise way based on the dimension function previously introduced. Thanks to this approximation and since reachability sets are almost semilinear we finally prove in Section 9 that the vector addition system reachability problem can be decided by inductive invariants definable in the Presburger arithmetic.

\section{Almost Semilinear Sets}

In this section we introduce the class of almost semilinear sets, a class of sets inspired by the geometrical characterization of the Presburger sets by semilinear sets.

We denote by $\mathbb{Z}, \mathbb{N}, \mathbb{N}_{>0}, \mathbb{Q}, \mathbb{Q} \geq 0, \mathbb{Q}>0$ the set of integers, natural numbers, positive integers, rational numbers, non negative rational numbers, and positive rational numbers. Vectors and sets of vectors are denoted in bold face. The $i$ th component of a vector $\boldsymbol{v} \in \mathbb{Q}^{d}$ is denoted by $\boldsymbol{v}(i)$. Given two sets $\boldsymbol{V}_{1}, \boldsymbol{V}_{2} \subseteq \mathbb{Q}^{d}$ we denote by $\boldsymbol{V}_{1}+\boldsymbol{V}_{2}$ the set $\left\{\boldsymbol{v}_{1}+\boldsymbol{v}_{2} \mid\left(\boldsymbol{v}_{1}, \boldsymbol{v}_{2}\right) \in \boldsymbol{V}_{1} \times \boldsymbol{V}_{2}\right\}$, and we denote by $\boldsymbol{V}_{1}-\boldsymbol{V}_{2}$ the set $\left\{\boldsymbol{v}_{1}-\boldsymbol{v}_{2} \mid\left(\boldsymbol{v}_{1}, \boldsymbol{v}_{2}\right) \in \boldsymbol{V}_{1} \times \boldsymbol{V}_{2}\right\}$. Given $T \subseteq \mathbb{Q}$ and $\boldsymbol{V} \subseteq \mathbb{Q}^{d}$ we let $T \boldsymbol{V}=\{t \boldsymbol{v} \mid(t, \boldsymbol{v}) \in T \times \boldsymbol{V}\}$. We also denote by $\boldsymbol{v}_{1}+\boldsymbol{V}_{2}$ and $\boldsymbol{V}_{1}+\boldsymbol{v}_{2}$ the sets $\left\{\boldsymbol{v}_{1}\right\}+\boldsymbol{V}_{2}$ and $\boldsymbol{V}_{1}+\left\{\boldsymbol{v}_{2}\right\}$, and we denote by $t \boldsymbol{V}$ and $T \boldsymbol{v}$ the sets $\{t\} \boldsymbol{V}$ and $T\{\boldsymbol{v}\}$.

A periodic set is a subset $\boldsymbol{P} \subseteq \mathbb{Z}^{d}$ such that $\mathbf{0} \in \boldsymbol{P}$ and $\boldsymbol{P}+\boldsymbol{P} \subseteq \boldsymbol{P}$. A conic set is a subset $\boldsymbol{C} \subseteq \mathbb{Q}^{d}$ such that $\mathbf{0} \in \boldsymbol{C}, \boldsymbol{C}+\boldsymbol{C} \subseteq \boldsymbol{C}$ and $\mathbb{Q}_{\geq 0} \boldsymbol{C} \subseteq \boldsymbol{C}$. A periodic set $\boldsymbol{P}$ is said to be finitely generated if there exist vectors $\boldsymbol{p}_{1}, \ldots, \boldsymbol{p}_{k} \in \boldsymbol{P}$ such that $\boldsymbol{P}=\mathbb{N} \boldsymbol{p}_{1}+\cdots+\mathbb{N} \boldsymbol{p}_{k}$. A periodic set $\boldsymbol{P}$ is said to be asymptotically definable if the conic set $\mathbb{Q}_{\geq 0} \boldsymbol{P}$ is definable in FO $(\mathbb{Q},+, \leq)$. Observe that finitely generated periodic sets are asymptotically definable since the conic set $\mathbb{Q}_{\geq 0} \boldsymbol{P}$ generated by $\boldsymbol{P}=\mathbb{N} \boldsymbol{p}_{1}+\cdots+\mathbb{N} \boldsymbol{p}_{k}$ is equal to $\mathbb{Q}_{\geq 0} \boldsymbol{p}_{1}+\cdots+\mathbb{Q}_{\geq 0} \boldsymbol{p}_{k}$. 
Example 2.1. The periodic set $\boldsymbol{P}=\{\boldsymbol{p} \in$ $\left.\mathbb{N}^{2} \mid \boldsymbol{p}(2) \leq \boldsymbol{p}(1) \leq 2^{\boldsymbol{p}(2)}-1\right\}$ is depicted on the right. Observe that $\mathbb{Q} \geq 0 \boldsymbol{P}$ is the conic set $\{\mathbf{0}\} \cup\left\{\boldsymbol{c} \in \mathbb{Q}_{>0}^{2} \mid \boldsymbol{c}(2) \leq \boldsymbol{c}(1)\right\}$ which is definable in $\mathrm{FO}(\mathbb{Q},+, \leq)$.

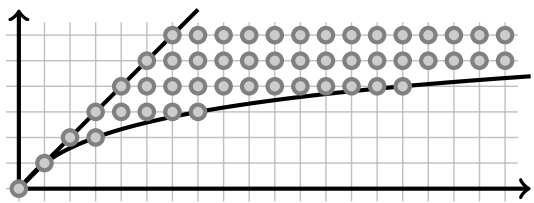

A Presburger set is a set $\boldsymbol{Z} \subseteq \mathbb{Z}^{d}$ definable in $\mathrm{FO}(\mathbb{Z},+, \leq)$. Recall that $\boldsymbol{Z} \subseteq \mathbb{Z}^{d}$ is a Presburger set iff it is semilinear, i.e. a finite union of linear sets $\boldsymbol{b}+\boldsymbol{P}$ where $\boldsymbol{b} \in \mathbb{Z}^{d}$ and $\boldsymbol{P} \subseteq \mathbb{Z}^{d}$ is a finitely generated periodic set [3]. The class of almost semilinear sets [10] is obtained from the definition of semilinear sets by weakening the finiteness condition on the considered periodic sets. More formally, an almost semilinear set is a finite union of sets of the form $\boldsymbol{b}+\boldsymbol{P}$ where $\boldsymbol{b} \in \mathbb{Z}^{d}$ and $\boldsymbol{P} \subseteq \mathbb{Z}^{d}$ is an asymptotically definable periodic set.

\section{Vector Addition Systems}

A Vector Addition System (VAS) is given by a finite subset $\boldsymbol{A} \subseteq \mathbb{Z}^{d}$. A vector $\boldsymbol{a} \in \boldsymbol{A}$ is called an action. A configuration is a vector $\boldsymbol{c} \in \mathbb{N}^{d}$. A run $\rho$ is a non-empty word $\rho=\boldsymbol{c}_{0} \ldots \boldsymbol{c}_{k}$ of configurations such that the difference $\boldsymbol{a}_{j}=\boldsymbol{c}_{j}-\boldsymbol{c}_{j-1}$ is in $\boldsymbol{A}$ for every $j \in\{1, \ldots, k\}$. In that case we say that $\rho$ is labeled by $w=\boldsymbol{a}_{1} \ldots \boldsymbol{a}_{k}$, the configurations $\boldsymbol{c}_{0}$ and $\boldsymbol{c}_{k}$ are respectively called the source and the target and they are denoted by $\operatorname{src}(\rho)$ and $\operatorname{tgt}(\rho)$. The direction of $\rho$ is the pair $(\operatorname{src}(\rho), \operatorname{tgt}(\rho))$, denoted by $\operatorname{dir}(\rho)$. Given a word $w \in \boldsymbol{A}^{*}$, we introduce the binary relation $\stackrel{w}{\longrightarrow}$ over the set of configurations by $\boldsymbol{x} \stackrel{w}{\longrightarrow} \boldsymbol{y}$ if there exists a run $\rho$ from $\boldsymbol{x}$ to $\boldsymbol{y}$ labeled by $w$. Observe that in this case $\rho$ is unique. The displacement of a word $w=\boldsymbol{a}_{1} \ldots \boldsymbol{a}_{k}$ of actions $\boldsymbol{a}_{j} \in \boldsymbol{A}$ is the vector $\Delta(w)=\sum_{j=1}^{k} \boldsymbol{a}_{j}$. Note that $\boldsymbol{x} \stackrel{w}{\rightarrow} \boldsymbol{y}$ implies $\boldsymbol{x}+\Delta(w)=\boldsymbol{y}$ but the converse is not true in general. The reachability relation is the relation $\stackrel{*}{\rightarrow}$ over $\mathbb{N}^{d}$ defined by $\boldsymbol{x} \stackrel{*}{\rightarrow} \boldsymbol{y}$ if there exists a run from $\boldsymbol{x}$ to $\boldsymbol{y}$. The following simple lemma is central in this paper.

Lemma 3.1 (Monotony). We have $\boldsymbol{c}+\boldsymbol{x} \stackrel{w}{\rightarrow} \boldsymbol{c}+\boldsymbol{y}$ for every $\boldsymbol{x} \stackrel{w}{\rightarrow} \boldsymbol{y}$ and for every $\boldsymbol{c} \in \mathbb{N}^{d}$.

Proof. Just observe that if $\rho=\boldsymbol{c}_{1} \ldots \boldsymbol{c}_{k}$ is a run from $\boldsymbol{x}$ to $\boldsymbol{y}$ labeled by $w$ where $\boldsymbol{c}_{j} \in \mathbb{N}^{d}$ then $\rho^{\prime}=\boldsymbol{c}_{1}^{\prime} \ldots \boldsymbol{c}_{k}^{\prime}$ where $\boldsymbol{c}_{j}^{\prime}=\boldsymbol{c}+\boldsymbol{c}_{j}$ is a run from $\boldsymbol{c}+\boldsymbol{x}$ to $\boldsymbol{c}+\boldsymbol{y}$ labeled by $w$.

The set of configurations forward reachable from a configuration $\boldsymbol{x} \in \mathbb{N}^{d}$ is the set $\left\{\boldsymbol{c} \in \mathbb{N}^{d} \mid\right.$ $\boldsymbol{x} \stackrel{*}{\rightarrow} \boldsymbol{c}\}$ denoted by $\operatorname{post}^{*}(\boldsymbol{x})$. Symmetrically the set of configurations backward reachable from a configuration $\boldsymbol{y} \in \mathbb{N}^{d}$ is the set $\left\{\boldsymbol{c} \in \mathbb{N}^{d} \mid \boldsymbol{c} \stackrel{*}{\rightarrow} \boldsymbol{y}\right\}$ denoted by $\operatorname{pre}^{*}(\boldsymbol{y})$. These definitions are extended over sets of configurations $\boldsymbol{X}, \boldsymbol{Y} \subseteq \mathbb{N}^{d}$ by $\operatorname{post}^{*}(\boldsymbol{X})=\bigcup_{\boldsymbol{x} \in \boldsymbol{X}} \operatorname{post}^{*}(\boldsymbol{x})$ and $\operatorname{pre}^{*}(\boldsymbol{Y})=$ $\bigcup_{\boldsymbol{y} \in \boldsymbol{Y}} \operatorname{post}^{*}(\boldsymbol{y})$. A set $\boldsymbol{X} \subseteq \mathbb{N}^{d}$ is said to be a forward inductive invariant if $\boldsymbol{X}=\operatorname{post}^{*}(\boldsymbol{X})$. Symmetrically a set $\boldsymbol{Y} \subseteq \mathbb{N}^{d}$ is said to be a backward inductive invariant if $\boldsymbol{Y}=\operatorname{pre}^{*}(\boldsymbol{Y})$.

In this paper we prove that for every $\boldsymbol{x}, \boldsymbol{y} \in \mathbb{N}^{d}$ such that there does not exist a run from $\boldsymbol{x}$ to $\boldsymbol{y}$, then there exists a pair $(\boldsymbol{X}, \boldsymbol{Y})$ of disjoint Presburger sets $\boldsymbol{X}, \boldsymbol{Y} \subseteq \mathbb{N}^{d}$ such that $\boldsymbol{X}$ is a forward inductive invariant that contains $\boldsymbol{x}$ and $\boldsymbol{Y}$ is a backward inductive invariant that contains $\boldsymbol{y}$. This result will provide directly the following theorem.

Theorem 3.2. The reachability problem for vector addition systems is decidable.

Proof. Let $\boldsymbol{x}, \boldsymbol{y} \in \mathbb{N}^{d}$ be two configurations. Let us consider an algorithm that enumerates in parallel the runs $\rho$ and the pairs $(\boldsymbol{X}, \boldsymbol{Y})$ of disjoint Presburger sets $\boldsymbol{X}, \boldsymbol{Y} \subseteq \mathbb{N}^{d}$ thanks to formulas in the Presburger arithmetic $\mathrm{FO}(\mathbb{Z},+, \leq)$. If the algorithm encounters a run from $\boldsymbol{x}$ to $\boldsymbol{y}$ then it returns "reachable" and if $\boldsymbol{X}$ is a forward inductive invariant that contains $\boldsymbol{x}$ 
and $\boldsymbol{Y}$ is a backward inductive invariant that contains $\boldsymbol{y}$ then it returns "unreachable". This last condition can be effectively decided as follows. Note that a set $\boldsymbol{X} \subseteq \mathbb{N}^{d}$ is a forward inductive invariant iff the set $\mathbb{N}^{d} \cap(\boldsymbol{X}+\boldsymbol{A}) \backslash \boldsymbol{X}$ denoted by $\tilde{\boldsymbol{X}}$ is empty, and a set $\boldsymbol{Y} \subseteq \mathbb{N}^{d}$ is a backward inductive invariant iff the set $\mathbb{N}^{d} \cap(\boldsymbol{Y}-\boldsymbol{A}) \backslash \boldsymbol{Y}$ denoted by $\tilde{\boldsymbol{Y}}$ is empty. Moreover, from Presburger formulas denoting $\boldsymbol{X}$ and $\boldsymbol{Y}$ we compute in linear time formulas denoting the sets $\tilde{\boldsymbol{X}}$ and $\tilde{\boldsymbol{Y}}$. Hence deciding that $\boldsymbol{X}$ is a forward inductive invariant that contains $\boldsymbol{x}$ and $\boldsymbol{Y}$ is a backward inductive invariant that contains $\boldsymbol{y}$ reduces to the satisfiability of formulas in the Presburger arithmetic. Since this logic is decidable, we deduce a way for implementing the last condition of our algorithm. Note that this algorithm is correct. Moreover, it terminates thanks to the main result proved in this paper.

Remark 3.3. The set post* $(\boldsymbol{x})$ is a forward inductive invariant that contains $\boldsymbol{x}$ and $\operatorname{pre}^{*}(\boldsymbol{y})$ is a backward inductive invariant that contains $\boldsymbol{y}$. Moreover, if there does not exist a run from $\boldsymbol{x}$ to $\boldsymbol{y}$ then these two reachability sets are disjoint. However in general reachability sets are not definable in the Presburger arithmetic [4].

\section{Well-Order Over The Runs}

An order $\sqsubseteq$ over a set $S$ is said to be a well-order if for every sequence $\left(s_{j}\right)_{j \in \mathbb{N}}$ of elements $s_{j} \in S$ there exist $j<k$ such that $s_{j} \sqsubseteq s_{k}$. Observe that $(\mathbb{N}, \leq)$ is a well-ordered set whereas $(\mathbb{Z}, \leq)$ is not well-ordered. As another example, the pigeon-hole principle shows that a set $S$ is well-ordered by the equality relation if and only if $S$ is finite. Well-orders can be easily defined thanks to Dickson's lemma and Higman's lemma as follows.

Dickson's lemma: Dickson's lemma shows that the cartesian product of two well-ordered sets is well-ordered. More formally, given two ordered sets $\left(S_{1}, \sqsubseteq_{1}\right)$ and $\left(S_{2}, \sqsubseteq_{2}\right)$ we denote by $\sqsubseteq_{1} \times \sqsubseteq_{2}$ the order defined component-wise over the cartesian product $S_{1} \times S_{2}$ by $\left(s_{1}, s_{2}\right) \sqsubseteq_{1}$ $\times \sqsubseteq_{2}\left(s_{1}^{\prime}, s_{2}^{\prime}\right)$ if $s_{1} \sqsubseteq_{1} s_{1}^{\prime}$ and $s_{2} \sqsubseteq_{2} s_{2}^{\prime}$. Dickson's lemma says that $\left(S_{1} \times S_{2}, \sqsubseteq_{1} \times \sqsubseteq_{2}\right)$ is well-ordered for every well-ordered sets $\left(S_{1}, \sqsubseteq_{1}\right)$ and $\left(S_{2}, \sqsubseteq_{2}\right)$. As a direct application, the set $\mathbb{N}^{d}$ equipped with the component-wise extension of $\leq$ is well-ordered.

Higman's lemma: Higman's lemma shows that words over well-ordered alphabets can be well-ordered. More formally, given an ordered set $(S, \sqsubseteq)$, we introduce the set $S^{*}$ of words over $S$ equipped with the order $\sqsubseteq^{*}$ defined by $w \sqsubseteq^{*} w^{\prime}$ if $w$ and $w^{\prime}$ can be decomposed into $w=s_{1} \ldots s_{k}$ and $w^{\prime} \in S^{*} s_{1}^{\prime} S^{*} \ldots s_{k}^{\prime} S^{*}$ where $s_{j} \sqsubseteq s_{j}^{\prime}$ are in $S$ for every $j \in\{1, \ldots, k\}$. Higman's lemma says that $\left(S^{*}, \sqsubseteq^{*}\right)$ is well-ordered for every well-ordered set $(S, \sqsubseteq)$. As a classical application, the set of words over a finite alphabet $S$ is well-ordered by the sub-word relation $=^{*}$.

We define a well-order over the runs as follows. We introduce the relation $\unlhd$ over the runs defined by $\rho \unlhd \rho^{\prime}$ if $\rho$ is a run of the form $\rho=\boldsymbol{c}_{0} \ldots \boldsymbol{c}_{k}$ where $\boldsymbol{c}_{j} \in \mathbb{N}^{d}$ and if there exists a sequence $\left(\boldsymbol{v}_{j}\right)_{0 \leq j \leq k+1}$ of vectors $\boldsymbol{v}_{j} \in \mathbb{N}^{d}$ such that $\rho^{\prime}$ is a run of the form $\rho^{\prime}=\rho_{0} \ldots \rho_{k}$ where $\rho_{j}$ is a run from $\boldsymbol{c}_{j}+\boldsymbol{v}_{j}$ to $\boldsymbol{c}_{j}+\boldsymbol{v}_{j+1}$.

Lemma 4.1. The relation $\unlhd$ is a well-order over the runs.

Proof. A proof of this lemma with different notations can be obtained from Section 6 of [5] with a simple reduction. For sake of completeness, we prefer to give a direct proof of this important result. To do so, we introduce a well-order $\preceq$ over the runs based on Dickson's lemma and Higman's lemma and we show that $\preceq$ and $\unlhd$ are equal. We first associate to a run $\rho=\boldsymbol{c}_{0} \ldots \boldsymbol{c}_{k}$ the word $\alpha(\rho)=\left(\boldsymbol{a}_{1}, \boldsymbol{c}_{1}\right) \ldots\left(\boldsymbol{a}_{k}, \boldsymbol{c}_{k}\right)$ over the set $S=\boldsymbol{A} \times \mathbb{N}^{d}$ where $\boldsymbol{a}_{j}=\boldsymbol{c}_{j}-\boldsymbol{c}_{j-1}$. The set $S$ is well-ordered by the relation $\sqsubseteq$ defined by $\left(\boldsymbol{a}_{1}, \boldsymbol{c}_{1}\right) \sqsubseteq\left(\boldsymbol{a}_{2}, \boldsymbol{c}_{2}\right)$ if $\boldsymbol{a}_{1}=\boldsymbol{a}_{2}$ and $\boldsymbol{c}_{1} \leq \boldsymbol{c}_{2}$. Dickson's 
lemma shows that $\sqsubseteq$ is a well-order. The set of words $S^{*}$ is well-ordered thanks to Higman's lemma by the relation ${ }^{*}$. The well-order $\preceq$ over the runs is defined by $\rho \preceq \rho^{\prime}$ if $\operatorname{dir}(\rho) \leq \operatorname{dir}\left(\rho^{\prime}\right)$ and $\alpha(\rho) \sqsubseteq^{*} \alpha\left(\rho^{\prime}\right)$. Now, let us prove that $\preceq$ and $\unlhd$ are equal. We consider a run $\rho=\boldsymbol{c}_{0} \ldots \boldsymbol{c}_{k}$ with $\boldsymbol{c}_{j} \in \mathbb{N}^{d}$ and we introduce the action $\boldsymbol{a}_{j}=\boldsymbol{c}_{j}-\boldsymbol{c}_{j-1}$ for each $j \in\{1, \ldots, k\}$.

Assume first that $\rho \preceq \rho^{\prime}$ for some run $\rho^{\prime}$. Since $\alpha(\rho)=\left(\boldsymbol{a}_{1}, \boldsymbol{c}_{1}\right) \ldots\left(\boldsymbol{a}_{k}, \boldsymbol{c}_{k}\right)$ and $\alpha(\rho) \sqsubseteq^{*}$ $\alpha\left(\rho^{\prime}\right)$ we deduce a decomposition of $\alpha\left(\rho^{\prime}\right)$ into the following word where $\boldsymbol{c}_{j}^{\prime} \geq \boldsymbol{c}_{j}$ for every $j \in\{1, \ldots, k\}$ and $w_{0}, \ldots, w_{k} \in S^{*}$ :

$$
\alpha\left(\rho^{\prime}\right)=w_{0}\left(\boldsymbol{a}_{1}, \boldsymbol{c}_{1}^{\prime}\right) w_{1} \ldots\left(\boldsymbol{a}_{k}, \boldsymbol{c}_{k}^{\prime}\right) w_{k}
$$

In particular $\rho^{\prime}$ can be decomposed in $\rho^{\prime}=\rho_{0} \ldots \rho_{k}$ where $\rho_{0}$ is a run from $\operatorname{src}\left(\rho^{\prime}\right)$ to $\boldsymbol{c}_{1}^{\prime}-\boldsymbol{a}_{1}, \rho_{j}$ is a run from $\boldsymbol{c}_{j}^{\prime}$ to $\boldsymbol{c}_{j+1}^{\prime}-\boldsymbol{a}_{j+1}$ for every $j \in\{1, \ldots, k-1\}$, and $\rho_{k}$ is a run from $\boldsymbol{c}_{k}^{\prime} \operatorname{totgt}\left(\rho^{\prime}\right)$. Let us introduce the sequence $\left(\boldsymbol{v}_{j}\right)_{0 \leq j \leq k+1}$ of vectors defined by $\boldsymbol{v}_{0}=\operatorname{src}\left(\rho^{\prime}\right)-\operatorname{src}(\rho), \boldsymbol{v}_{j}=\boldsymbol{c}_{j}^{\prime}-\boldsymbol{c}_{j}$ for every $j \in\{1, \ldots, k\}$ and $\boldsymbol{v}_{k+1}=\operatorname{tgt}\left(\rho^{\prime}\right)-\operatorname{tgt}(\rho)$. Note that $\boldsymbol{v}_{j} \in \mathbb{N}^{d}$ for every $j \in\{0, \ldots, k+1\}$. Observe that for every $j \in\{1, \ldots, k-1\}$ we have $\boldsymbol{c}_{j+1}^{\prime}-\boldsymbol{a}_{j}=\boldsymbol{c}_{j+1}-\boldsymbol{a}_{j}+\boldsymbol{v}_{j+1}=\boldsymbol{c}_{j}+\boldsymbol{v}_{j+1}$. Hence $\rho_{j}$ is a run from $\boldsymbol{c}_{j}+\boldsymbol{v}_{j}$ to $\boldsymbol{c}_{j}+\boldsymbol{v}_{j+1}$ for every $j \in\{0, \ldots, k\}$. Therefore $\rho \unlhd \rho^{\prime}$.

Conversely, let us assume that $\rho \unlhd \rho^{\prime}$ for some run $\rho^{\prime}$. We introduce a sequence $\left(\boldsymbol{v}_{j}\right)_{0 \leq j \leq k+1}$ of vectors in $\mathbb{N}^{d}$ such that $\rho^{\prime}=\rho_{0} \ldots \rho_{k}$ where $\rho_{j}$ is a run from $\boldsymbol{c}_{j}+\boldsymbol{v}_{j}$ to $\boldsymbol{c}_{j}+\boldsymbol{v}_{j+1}$. We deduce the following equality where $\boldsymbol{a}_{j}^{\prime}=\operatorname{src}\left(\rho_{j}\right)-\operatorname{tgt}\left(\rho_{j-1}\right)$ :

$$
\alpha\left(\rho^{\prime}\right)=\alpha\left(\rho_{0}\right)\left(\boldsymbol{a}_{1}^{\prime}, \boldsymbol{c}_{1}+\boldsymbol{v}_{1}\right) \alpha\left(\rho_{1}\right) \ldots\left(\boldsymbol{a}_{k}^{\prime}, \boldsymbol{c}_{k}+\boldsymbol{v}_{k}\right) \alpha\left(\rho_{k}\right)
$$

Observe that $\boldsymbol{a}_{j}^{\prime}=\left(\boldsymbol{c}_{j}+\boldsymbol{v}_{j}\right)-\left(\boldsymbol{c}_{j-1}+\boldsymbol{v}_{j}\right)=\boldsymbol{a}_{j}$. We deduce that $\alpha(\rho) \sqsubseteq^{*} \alpha\left(\rho^{\prime}\right)$. Moreover, since $\operatorname{dir}(\rho) \leq \operatorname{dir}\left(\rho^{\prime}\right)$ we get $\rho \preceq \rho^{\prime}$.

\section{Transformer Relations}

Based on the definition of $\unlhd$, we introduce the transformer relation with capacity $c \in \mathbb{N}^{d}$ as the binary relation $\stackrel{c}{\curvearrowright}$ over $\mathbb{N}^{d}$ defined by $\boldsymbol{x} \stackrel{c}{\curvearrowright} \boldsymbol{y}$ if there exists a run from $\boldsymbol{c}+\boldsymbol{x}$ to $\boldsymbol{c}+\boldsymbol{y}$. We also associate to every run $\rho=\boldsymbol{c}_{0} \ldots \boldsymbol{c}_{k}$ with $\boldsymbol{c}_{j} \in \mathbb{N}^{d}$ the transformer relation along the run $\rho$ denoted by $\stackrel{\rho}{\curvearrowright}$ and defined as the following composition:

$$
\stackrel{\rho}{\curvearrowright}=\stackrel{c_{0}}{\curvearrowright} \circ \cdots \circ \stackrel{c_{k}}{\curvearrowright}
$$

In this section transformer relations are shown to be asymptotically definable periodic. Thanks to the following Lemma 5.1 , it is sufficient to prove that $\stackrel{c}{\curvearrowright}$ is in this class for every capacity $c \in \mathbb{N}^{d}$.

Lemma 5.1. Asymptotically definable periodic relations are stable by composition.

Proof. Assume that $R, S \subseteq \mathbb{Z}^{d} \times \mathbb{Z}^{d}$ are two periodic relations and observe that $(\mathbf{0}, \mathbf{0}) \in R \circ S$. Let us consider two pairs $\left(\boldsymbol{x}_{1}, \boldsymbol{z}_{1}\right)$ and $\left(\boldsymbol{x}_{2}, \boldsymbol{z}_{2}\right)$ in $R \circ S$. For each $k \in\{1,2\}$, there exists $\boldsymbol{y}_{k} \in \mathbb{Z}^{d}$ such that $\left(\boldsymbol{x}_{k}, \boldsymbol{y}_{k}\right) \in R$ and $\left(\boldsymbol{y}_{k}, \boldsymbol{z}_{k}\right) \in S$. As $R$ and $S$ are periodic we get $(\boldsymbol{x}, \boldsymbol{y}) \in R$ and $(\boldsymbol{y}, \boldsymbol{z}) \in S$ where $\boldsymbol{x}=\boldsymbol{x}_{1}+\boldsymbol{x}_{2}, \boldsymbol{y}=\boldsymbol{y}_{1}+\boldsymbol{y}_{2}$ and $\boldsymbol{z}=\boldsymbol{z}_{1}+\boldsymbol{z}_{2}$. Thus $(\boldsymbol{x}, \boldsymbol{z}) \in R \circ S$ and we have proved that $R \circ S$ is periodic. Now just observe that $\mathbb{Q}_{\geq 0}(R \circ S)=\left(\mathbb{Q}_{\geq 0} R\right) \circ\left(\mathbb{Q}_{\geq 0} S\right)$. Hence if $R$ and $S$ are asymptotically definable then $R \circ S$ is also asymptotically definable.

Lemma 5.2. The transformer relation $\stackrel{c}{\curvearrowright}$ is periodic.

Proof. Assume that $\boldsymbol{c}+\boldsymbol{x}_{1} \stackrel{w_{1}}{\longrightarrow} \boldsymbol{c}+\boldsymbol{y}_{1}$ and $\boldsymbol{c}+\boldsymbol{x}_{2} \stackrel{w_{2}}{\longrightarrow} \boldsymbol{c}+\boldsymbol{y}_{2}$ for words $w_{1}, w_{2} \in \boldsymbol{A}^{*}$ and vectors $\boldsymbol{x}_{1}, \boldsymbol{y}_{1}, \boldsymbol{x}_{2}, \boldsymbol{y}_{2} \in \mathbb{N}^{d}$. By monotony $\boldsymbol{c}+\boldsymbol{x}_{1}+\boldsymbol{x}_{2} \stackrel{w_{1} w_{2}}{\longrightarrow} \boldsymbol{c}+\boldsymbol{y}_{1}+\boldsymbol{y}_{2}$. 
In the remainder of this section, we show that $\mathbb{Q}_{\geq 0} \stackrel{c}{\curvearrowright}$ is definable in $\mathrm{FO}(\mathbb{Q},+, \leq)$. We introduce the set $\Gamma_{\boldsymbol{c}}$ of triples $\gamma=(\boldsymbol{x}, \boldsymbol{c}, \boldsymbol{y})$ such that $\boldsymbol{x} \stackrel{\boldsymbol{c}}{\curvearrowright} \boldsymbol{y}$ and the set $\Gamma=\bigcup_{\boldsymbol{c} \in \mathbb{N}^{d}} \Gamma_{\boldsymbol{c}}$. Given a triple $\gamma \in \Gamma$, the vectors $\boldsymbol{x}, \boldsymbol{c}, \boldsymbol{y}$ implicitly denote the components of $\gamma$. We introduce the set $\Omega_{\gamma}$ of runs $\rho$ such that $\operatorname{dir}(\rho) \in(\boldsymbol{c}, \boldsymbol{c})+\mathbb{N}(\boldsymbol{x}, \boldsymbol{y})$ and the set $\boldsymbol{Q}_{\gamma}$ of configurations $\boldsymbol{q} \in \mathbb{N}^{d}$ such that there exists a run $\rho \in \Omega_{\gamma}$ in which $\boldsymbol{q}$ occurs. We denote by $I_{\gamma}$ the set of indexes $i \in\{1, \ldots, d\}$ such that $\left\{\boldsymbol{q}(i) \mid \boldsymbol{q} \in \boldsymbol{Q}_{\gamma}\right\}$ is finite.

Example 5.3. Let us consider the VAS $\boldsymbol{A}=\{\boldsymbol{a}, \boldsymbol{b}\}$ where $\boldsymbol{a}=(1,1,-1)$ and $\boldsymbol{b}=(-1,0,1)$ and let $\gamma=$ $(\boldsymbol{x}, \boldsymbol{c}, \boldsymbol{y})$ where $\boldsymbol{x}=(0,0,0), \boldsymbol{c}=(1,0,1)$ and $\boldsymbol{y}=$ $(0,1,0)$. Since $\boldsymbol{x}=(0,0,0)$, we observe that $\Omega_{\gamma}=$ $\left\{\boldsymbol{c} \stackrel{w_{1} \ldots w_{n}}{\longrightarrow} \boldsymbol{c}+n \boldsymbol{y} \mid n \in \mathbb{N} w_{j} \in\{\boldsymbol{a b}, \boldsymbol{b a}\}\right\}$. This set of runs is depicted on the right. Observe that $\boldsymbol{Q}_{\gamma}=(\boldsymbol{c}+\boldsymbol{a}+\mathbb{N} \boldsymbol{y}) \cup(\boldsymbol{c}+\mathbb{N} \boldsymbol{y}) \cup(\boldsymbol{c}+\boldsymbol{b}+\mathbb{N} \boldsymbol{y})$. Hence the set of bounded components is $I_{\gamma}=\{1,3\}$.
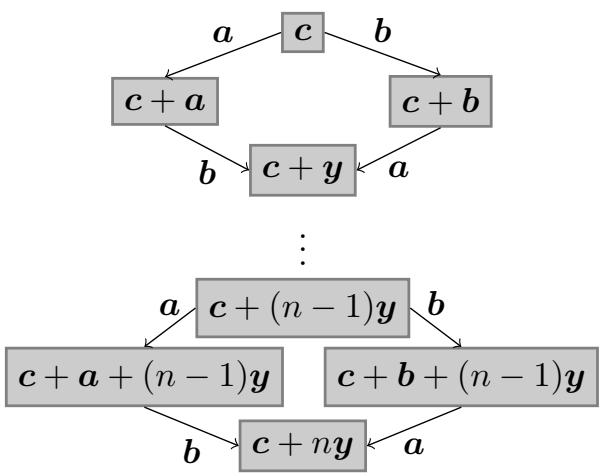

In section 5.1 we show that for every configuration $\boldsymbol{q} \in \boldsymbol{Q}_{\gamma}$, there exist configurations $\boldsymbol{q}^{\prime} \in \boldsymbol{Q}_{\gamma}$ that coincide with $\boldsymbol{q}$ on components indexed by $I_{\gamma}$ and such that $\boldsymbol{q}^{\prime}$ is as large as expected on all the other components. Based on a projection of the unbounded components of vectors in $\boldsymbol{Q}_{\gamma}$, i.e. the components not indexed by $I_{\gamma}$, we show in Section 5.3 that a finite graph $G_{\gamma}$ called production graph can be canonically associated to every triple $\gamma$. We also prove that the class $\left\{G_{\gamma} \mid \gamma \in \Gamma_{c}\right\}$ is finite. Finally in Section 5.2 we introduce a binary relation $R_{\gamma} \subseteq \mathbb{Q}_{\geq 0} \stackrel{c}{\curvearrowright}$ definable in $\mathrm{FO}(\mathbb{Q},+, \leq)$ associated to the production graphs $G_{\gamma}$ and such that $(\boldsymbol{x}, \boldsymbol{y}) \in R_{\gamma}$. By observing that $\mathbb{Q}_{\geq 0} \stackrel{c}{\curvearrowright}=\bigcup_{\gamma \in \Gamma_{c}} R_{\gamma}$ and the class $\left\{R_{\gamma} \mid \gamma \in \Gamma_{\boldsymbol{c}}\right\}$ is finite we deduce that the periodic relation $\stackrel{c}{\curvearrowright}$ is asymptotically definable.

\subsection{Intraproductions}

An intraproduction for $\gamma$ is a vector $\boldsymbol{h} \in \mathbb{N}^{d}$ such that there exists $n \in \mathbb{N}$ satisfying $n \boldsymbol{x} \stackrel{c}{\curvearrowright}$ $\boldsymbol{h} \stackrel{c}{\curvearrowright} n \boldsymbol{y}$. We denote by $\boldsymbol{H}_{\gamma}$ the set of intraproductions for $\gamma$. This set is periodic since $\stackrel{c}{\curvearrowright}$ is periodic. In particular for every $\boldsymbol{h} \in \boldsymbol{H}_{\gamma}$ we have $\mathbb{N} \boldsymbol{h} \subseteq \boldsymbol{H}_{\gamma}$ and the following lemma shows that $\boldsymbol{Q}_{\gamma}+\mathbb{N} \boldsymbol{h} \subseteq \boldsymbol{Q}_{\gamma}$. Hence, the components of every vector $\boldsymbol{q} \in \boldsymbol{Q}_{\gamma}$ indexed by $i$ such that $\boldsymbol{h}(i)>0$ can be increased to arbitrary large values by adding a large number of times the vector $\boldsymbol{h}$. In order to increase simultaneously all the components not indexed by $I_{\gamma}$ we are interested by intraproductions $\boldsymbol{h}$ such that $\boldsymbol{h}(i)>0$ for every $i \notin I_{\gamma}$. Note that components indexed by $I_{\gamma}$ are necessarily zero since for every intraproduction $\boldsymbol{h}$, from $\boldsymbol{c}+\mathbb{N} \boldsymbol{h} \subseteq \boldsymbol{Q}_{\gamma}$ we get $\boldsymbol{h}(i)=0$ for every $i \in I_{\gamma}$.

Example 5.4. Let us come back to Example 5.3. We have $\boldsymbol{H}_{\gamma}=\mathbb{N} \boldsymbol{y}$.

Lemma 5.5. We have $\boldsymbol{Q}_{\gamma}+\boldsymbol{H}_{\gamma} \subseteq \boldsymbol{Q}_{\gamma}$.

Proof. Let $\boldsymbol{q} \in \boldsymbol{Q}_{\gamma}$ and $\boldsymbol{h} \in \boldsymbol{H}_{\gamma}$. As $\boldsymbol{q} \in \boldsymbol{Q}_{\gamma}$, there exist $n \in \mathbb{N}$ and words $u, v \in \boldsymbol{A}^{*}$ such that $\boldsymbol{c}+n \boldsymbol{x} \stackrel{u}{\rightarrow} \boldsymbol{q} \stackrel{v}{\rightarrow} \boldsymbol{c}+n \boldsymbol{y}$. Since $\boldsymbol{h} \in \boldsymbol{H}_{\gamma}$ there exist $n^{\prime} \in \mathbb{N}$ and words $u^{\prime}, v^{\prime} \in \boldsymbol{A}^{*}$ such that $\boldsymbol{c}+n^{\prime} \boldsymbol{x} \stackrel{u^{\prime}}{\longrightarrow} \boldsymbol{c}+\boldsymbol{h} \stackrel{v^{\prime}}{\longrightarrow} \boldsymbol{c}+n^{\prime} \boldsymbol{y}$. Let $m=n+n^{\prime}$. By monotony, we have $\boldsymbol{c}+m \boldsymbol{x} \stackrel{u^{\prime} u}{\longrightarrow} \boldsymbol{q}+\boldsymbol{h} \stackrel{v v^{\prime}}{\longrightarrow}$ $\boldsymbol{c}+m \boldsymbol{y}$. Hence $\boldsymbol{q}+\boldsymbol{h} \in \boldsymbol{Q}_{\gamma}$. 
Lemma 5.6. For every $\boldsymbol{q} \leq \boldsymbol{q}^{\prime}$ in $\boldsymbol{Q}_{\gamma}$ there exists $\boldsymbol{h} \in \boldsymbol{H}_{\gamma}$ such that $\boldsymbol{q}^{\prime} \leq \boldsymbol{q}+\boldsymbol{h}$.

Proof. As $\boldsymbol{q}, \boldsymbol{q}^{\prime} \in \boldsymbol{Q}_{\gamma}$ there exists $m, m^{\prime} \in \mathbb{N}$ and $u, v, u^{\prime}, v^{\prime} \in \boldsymbol{A}^{*}$ such that:

$$
\boldsymbol{c}+m \boldsymbol{x} \stackrel{\leftrightarrow}{\rightarrow} \boldsymbol{q} \stackrel{v}{\rightarrow} \boldsymbol{c}+m \boldsymbol{y} \quad \text { and } \quad \boldsymbol{c}+m^{\prime} \boldsymbol{x} \stackrel{u^{\prime}}{\rightarrow} \boldsymbol{q}^{\prime} \stackrel{v^{\prime}}{\rightarrow} \boldsymbol{c}+m^{\prime} \boldsymbol{y}
$$

Let us introduce $\boldsymbol{v}=\boldsymbol{q}^{\prime}-\boldsymbol{q}, \boldsymbol{h}=\boldsymbol{v}+m(\boldsymbol{x}+\boldsymbol{y})$, and $n=m+m^{\prime}$. By monotony:

$$
\begin{aligned}
\boldsymbol{c}+n \boldsymbol{x} \stackrel{u^{\prime}}{\rightarrow} \boldsymbol{q}^{\prime}+m \boldsymbol{x} & \text { and } & \boldsymbol{q}+\boldsymbol{v}+m \boldsymbol{x} \stackrel{v}{\rightarrow} \boldsymbol{c}+\boldsymbol{h} \\
\boldsymbol{c}+\boldsymbol{h} \stackrel{u}{\rightarrow} \boldsymbol{q}+\boldsymbol{v}+m \boldsymbol{y} & \text { and } & \boldsymbol{q}^{\prime}+m \boldsymbol{y} \stackrel{v^{\prime}}{\rightarrow} \boldsymbol{c}+n \boldsymbol{y}
\end{aligned}
$$

Since $\boldsymbol{q}^{\prime}+m \boldsymbol{x}=\boldsymbol{q}+\boldsymbol{v}+m \boldsymbol{x}$ and $\boldsymbol{q}+\boldsymbol{v}+m \boldsymbol{y}=\boldsymbol{q}^{\prime}+m \boldsymbol{y}$, we have proved that $\boldsymbol{c}+n \boldsymbol{x} \stackrel{u^{\prime} v}{\longrightarrow}$ $\boldsymbol{c}+\boldsymbol{h} \stackrel{u v^{\prime}}{\longrightarrow} \boldsymbol{c}+n \boldsymbol{y}$. Hence $\boldsymbol{h} \in \boldsymbol{H}_{\gamma}$. Observe that $\boldsymbol{q}+\boldsymbol{h}=\boldsymbol{q}^{\prime}+m(\boldsymbol{x}+\boldsymbol{y}) \geq \boldsymbol{q}^{\prime}$. We are done.

Lemma 5.7. There exist $\boldsymbol{h} \in \boldsymbol{H}_{\gamma}$ such that $I_{\gamma}=\{i \mid \boldsymbol{h}(i)=0\}$.

Proof. Let $i \notin I_{\gamma}$. There exists a sequence $\left(\boldsymbol{q}_{j}\right)_{j \in \mathbb{N}}$ of configurations $\boldsymbol{q}_{j} \in \boldsymbol{Q}_{\gamma}$ such that $\left(\boldsymbol{q}_{j}(i)\right)_{j \in \mathbb{N}}$ is strictly increasing. Since $\left(\mathbb{N}^{d}, \leq\right)$ is well-ordered there exists $j<k$ such that $\boldsymbol{q}_{j} \leq \boldsymbol{q}_{k}$. Lemma 5.6 shows that there exists an intraproduction $\boldsymbol{h}_{i}$ for $\gamma$ such that $\boldsymbol{q}_{k} \leq \boldsymbol{q}_{j}+\boldsymbol{h}_{i}$. In particular $\boldsymbol{h}_{i}(i)>0$ since $\boldsymbol{q}_{j}(i)<\boldsymbol{q}_{k}(i)$. As the set of intraproductions $\boldsymbol{H}_{\gamma}$ is periodic we deduce that $\boldsymbol{h}=\sum_{i \notin I} \boldsymbol{h}_{i}$ is an intraproduction for $\gamma$. By construction we have $\boldsymbol{h}(i)>0$ for every $i \notin I_{\gamma}$. Since $\boldsymbol{h} \in \boldsymbol{H}_{\gamma}$ we deduce that $\boldsymbol{h}(i)=0$ for every $i \in I_{\gamma}$. Therefore $I_{\gamma}=\{i \mid \boldsymbol{h}(i)=0\}$.

\subsection{Production Graphs}

Finite graphs $G_{\gamma}$, called production graphs can be associated to every triple $\gamma$ as follows. The set of states is obtained from $\boldsymbol{Q}_{\gamma}$ by projecting away the unbounded components. More formally, we introduce the projection function $\pi_{\gamma}: \boldsymbol{Q}_{\gamma} \rightarrow \mathbb{N}^{I_{\gamma}}$ defined by $\pi_{\gamma}(\boldsymbol{q})(i)=\boldsymbol{q}(i)$ for every $\boldsymbol{q} \in \boldsymbol{Q}_{\gamma}$ and for every $i \in I_{\gamma}$. We consider the finite set of states $S_{\gamma}=\pi_{\gamma}\left(\boldsymbol{Q}_{\gamma}\right)$ and the set $T_{\gamma}$ of transitions $\left(\pi_{\gamma}(\boldsymbol{q}), \boldsymbol{q}^{\prime}-\boldsymbol{q}, \pi_{\gamma}\left(\boldsymbol{q}^{\prime}\right)\right)$ where $\boldsymbol{q} \boldsymbol{q}^{\prime}$ is a factor of a run in $\Omega_{\gamma}$. Since $T_{\gamma} \subseteq S_{\gamma} \times \boldsymbol{A} \times S_{\gamma}$ we deduce that $T_{\gamma}$ is finite. We introduce the finite graph $G_{\gamma}=\left(S_{\gamma}, T_{\gamma}\right)$, called the production graph of $\gamma$. Since $\boldsymbol{c} \in \boldsymbol{Q}_{\gamma}$ we deduce that $\pi_{\gamma}(\boldsymbol{c})$ is a state of $G_{\gamma}$. This state, called the special state for $\gamma$, is denoted by $s_{\gamma}$.

Example 5.8. Let us come back to Example 5.3 Observe that $\pi_{\gamma}(\boldsymbol{c}+\boldsymbol{a}+n \boldsymbol{y})=(2, \star, 0), \pi_{\gamma}(\boldsymbol{c}+n \boldsymbol{y})=$ $(1, \star, 1)$, and $\pi_{\gamma}(\boldsymbol{c}+\boldsymbol{b}+n \boldsymbol{y})=(0, \star, 2)$ where $\star$ denotes a projected component. The graph $G_{\gamma}$ is depicted on the right. Note that $s_{\gamma}=(1, \star, 1)$.

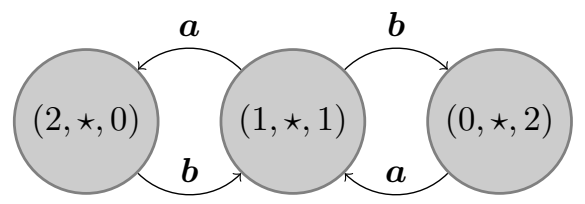

Corollary 5.9. We have $\pi_{\gamma}(\operatorname{src}(\rho))=s_{\gamma}=\pi_{\gamma}(\operatorname{tgt}(\rho))$ for every run $\rho \in \Omega_{\gamma}$.

Proof. Since $\rho \in \Omega_{\gamma}$ there exists $n \in \mathbb{N}$ such that $\rho$ is a run from $\boldsymbol{c}+n \boldsymbol{x}$ to $\boldsymbol{c}+n \boldsymbol{y}$. In particular $n \boldsymbol{x}$ and $n \boldsymbol{y}$ are two intraproductions for $\gamma$. We get $n \boldsymbol{x}(i)=0=n \boldsymbol{y}(i)$ for every $i \in I_{\gamma}$. Hence $\pi_{\gamma}(\operatorname{src}(\rho))=\pi_{\gamma}(\boldsymbol{c})=\pi_{\gamma}(\operatorname{tgt}(\rho))$.

A path in $G_{\gamma}$ is a word $p=\left(s_{0}, \boldsymbol{a}_{1}, s_{1}\right) \ldots\left(s_{k-1}, \boldsymbol{a}_{k}, s_{k}\right)$ of transitions $\left(s_{j-1}, \boldsymbol{a}_{j}, s_{j}\right)$ in $T_{\gamma}$. Such a path is called a path from $s_{0}$ to $s_{k}$ labeled by $w=\boldsymbol{a}_{1} \ldots \boldsymbol{a}_{k}$. When $s_{0}=s_{k}$ the path is 
called a cycle. The previous corollary shows that for every run $\rho=\boldsymbol{c}_{0} \ldots \boldsymbol{c}_{k}$ in $\Omega_{\gamma}$ the following word $\theta_{\rho}$ is a cycle on $s_{\gamma}$ in $G_{\gamma}$ labeled by $w$ :

$$
\theta_{\rho}=\left(\pi_{\gamma}\left(\boldsymbol{c}_{0}\right), \boldsymbol{a}_{1}, \pi_{\gamma}\left(\boldsymbol{c}_{1}\right)\right) \ldots\left(\pi_{\gamma}\left(\boldsymbol{c}_{k-1}\right), \boldsymbol{a}_{k}, \pi_{\gamma}\left(\boldsymbol{c}_{k}\right)\right)
$$

Corollary 5.10. The graph $G_{\gamma}$ is strongly connected.

Proof. Let $s \in S_{\gamma}$. There exists $\boldsymbol{q} \in \boldsymbol{Q}_{\gamma}$ that occurs in a run $\rho \in \Omega_{\gamma}$ such that $s=\pi_{\gamma}(\boldsymbol{q})$. Hence there exist $u, v \in \boldsymbol{A}^{*}$ such that $\operatorname{src}(\rho) \stackrel{u}{\rightarrow} \boldsymbol{q} \stackrel{v}{\rightarrow} \operatorname{tgt}(\rho)$. Note that $\theta_{\rho}$ is the concatenation of a path from $s_{\gamma}$ to $s$ and a path from $s$ to $s_{\gamma}$ labeled by $u, v$.

Corollary 5.11. States in $S_{\gamma}$ are incomparable.

Proof. Let us consider $s \leq s^{\prime}$ in $S_{\gamma}$. There exists $\boldsymbol{q}, \boldsymbol{q}^{\prime} \in \boldsymbol{Q}_{\gamma}$ such that $s=\pi_{\gamma}(\boldsymbol{q})$ and $s^{\prime}=\pi_{\gamma}\left(\boldsymbol{q}^{\prime}\right)$. Lemma 5.7 shows that there exists an intraproduction $\boldsymbol{h}^{\prime} \in \boldsymbol{H}_{\gamma}$ such that $I_{\gamma}=\left\{i \mid \boldsymbol{h}^{\prime}(i)=0\right\}$. By replacing $\boldsymbol{h}^{\prime}$ by a vector in $\mathbb{N}_{>0} \boldsymbol{h}^{\prime}$ we can assume without loss of generality that $\boldsymbol{q}(i) \leq$ $\boldsymbol{q}^{\prime}(i)+\boldsymbol{h}^{\prime}(i)$ for every $i \notin I_{\gamma}$. As $\boldsymbol{q}(i)=s(i) \leq s^{\prime}(i)=\boldsymbol{q}^{\prime}(i)=\boldsymbol{q}^{\prime}(i)+\boldsymbol{h}^{\prime}(i)$ for every $i \in I_{\gamma}$ we deduce that $\boldsymbol{q} \leq \boldsymbol{q}^{\prime}+\boldsymbol{h}^{\prime}$. Lemma 5.5 shows that $\boldsymbol{q}^{\prime}+\boldsymbol{h}^{\prime} \in \boldsymbol{Q}_{\gamma}$. Lemma 5.6 shows that there exists an intraproduction $\boldsymbol{h} \in \boldsymbol{H}_{\gamma}$ such that $\boldsymbol{q}^{\prime}+\boldsymbol{h}^{\prime} \leq \boldsymbol{q}+\boldsymbol{h}$. As $\boldsymbol{h} \in \boldsymbol{H}_{\gamma}$ we deduce that $\boldsymbol{h}(i)=0$ for every $i \in I_{\gamma}$. In particular $\boldsymbol{q}^{\prime}(i) \leq \boldsymbol{q}(i)$ for every $i \in I_{\gamma}$. Hence $s^{\prime} \leq s$ and we get $s=s^{\prime}$.

Corollary 5.12. The class $\left\{G_{\gamma} \mid \gamma \in \Gamma_{\boldsymbol{c}}\right\}$ is finite.

Proof. Given $I \subseteq\{1, \ldots, d\}$ we introduce the state $s_{\boldsymbol{c}, I} \in \mathbb{N}^{I}$ defined by $s_{\boldsymbol{c}, I}(i)=\boldsymbol{c}(i)$ for every $i \in I$. We also introduce the set $\Gamma_{c, I}$ of triples $\gamma \in \Gamma_{\boldsymbol{c}}$ such that $I_{\gamma}=I$. Note that in this case $s_{c, I}$ is equal to the special state $s_{\gamma}$ for $\gamma$. Assume by contradiction that $S_{c, I}=\bigcup_{\gamma \in \Gamma_{c, I}} S_{\gamma}$ is infinite. For every $s \in S_{c, I}$ there exists $\gamma \in \Gamma_{\boldsymbol{c}, I}$ such that $s \in S_{\gamma}$. Hence there exists a path $p_{s}$ in $G_{\gamma}$ from $s_{c, I}$ to $s$. Since the states in $S_{\gamma}$ are incomparable, we can assume that the states occurring in $p_{s}$ are incomparable. By inserting the paths $p_{s}$ in a tree rooted by $s_{\boldsymbol{c}, I}$ with transitions labeled by actions in $\boldsymbol{A}$ we deduce an infinite tree such that each node has a finite number of children (at most $|\boldsymbol{A}|$ ). Koenig's lemma shows that this tree has an infinite branch. Since $\left(\mathbb{N}^{I}, \leq\right)$ is well-ordered, there exists two comparable distinct nodes in this branch. There exists $s \in S_{c, I}$ such that these two comparable states occurs in $p_{s}$. We get a contradiction. Thus $S_{c, I}$ is finite. We deduce the corollary.

\subsection{Kirchhoff's Functions}

We associate to the production graph $G_{\gamma}$ a binary relation $R_{\gamma}$ included in $\mathbb{Q}_{\geq 0} \stackrel{c}{\curvearrowright}$ and such that $(\boldsymbol{x}, \boldsymbol{y}) \in R_{\gamma}$. This relation is based on Kirchhoff's functions.

A Kirchhoff's function for $\gamma$ is a function $f: T_{\gamma} \rightarrow \mathbb{Q}$ labeling transitions of the production graph $G_{\gamma}$ by rational numbers satisfying the following equality for every $s \in S_{\gamma}$ :

$$
\sum_{t \in T_{\gamma} \cap\left(\{s\} \times \boldsymbol{A} \times S_{\gamma}\right)} f(t)=\sum_{t \in T_{\gamma} \cap\left(S_{\gamma} \times \boldsymbol{A} \times\{s\}\right)} f(t)
$$

Kirchhoff's functions $f: T_{\gamma} \rightarrow \mathbb{N}_{>0}$ are characterized as follows. A cycle $\theta$ in $G_{\gamma}$ is said to be total for $\gamma$ if every transition in $T_{\gamma}$ occurs in $\theta$. The Parikh image of a path is the function $f: T_{\gamma} \rightarrow \mathbb{N}$ where $f(t)$ denotes the number of occurrences of $t$ in the path. Since $G_{\gamma}$ is strongly 
connected, Euler's lemma shows that a function $f: T_{\gamma} \rightarrow \mathbb{N}_{>0}$ is a Kirchhoff' function for $\gamma$ if and only if $f$ is the Parikh image of a total cycle for $\gamma$.

The displacement of a function $f: T_{\gamma} \rightarrow \mathbb{Q}$ is the sum $\sum_{t \in T_{\gamma}} f(t) \Delta(t)$ where $\Delta(t)=\boldsymbol{a}$ if $\boldsymbol{a}$ is the label of the transition $t$. This displacement is denoted by $\Delta(f)$. Let us observe that if $f$ is the Parikh's image of a path in $G_{\gamma}$ labeled by a word $w$ then $\Delta(f)=\Delta(w)$. Intuitively the displacement of $w$ only depends on the number of times transitions in $T_{\gamma}$ occur in the path.

We introduce the relation $R_{\gamma}$ of pairs $(\boldsymbol{u}, \boldsymbol{v}) \in \mathbb{Q}_{\geq 0}^{d} \times \mathbb{Q}_{\geq 0}^{d}$ satisfying $\boldsymbol{u}(i)>0$ iff $\boldsymbol{x}(i)>0$, $\boldsymbol{v}(i)>0$ iff $\boldsymbol{y}(i)>0$, and such that there exists a Kirchhoff's function $f: T_{\gamma} \rightarrow \mathbb{Q}_{>0}$ such that $\boldsymbol{v}-\boldsymbol{u}=\Delta(f)$. Observe that $R_{\gamma}$ is definable in $\mathrm{FO}(\mathbb{Q},+, \leq)$.

Example 5.13. Let us come back to Examples 5.3 and 5.8. A function $f: T_{\gamma} \rightarrow \mathbb{Q}$ is a Kirchhoff's function for $\gamma$ if and only if $f((1, \star, 1), \boldsymbol{a},(2, \star, 0))=f((2, \star, 0), \boldsymbol{b},(1, \star, 1))$ and $f((1, \star, 1), \boldsymbol{b},(0, \star, 2))=f((0, \star, 2), \boldsymbol{a},(1, \star, 1))$. We get $R_{\gamma}=\left\{((0,0,0),(0, n, 0)) \mid n \in \mathbb{Q}_{>0}\right\}$.

Lemma 5.14. We have $(\boldsymbol{x}, \boldsymbol{y}) \in R_{\gamma}$.

Proof. Assume that $T_{\gamma}=\left\{t_{1}, \ldots, t_{k}\right\}$. By definition of $T_{\gamma}$, for every $j \in\{1, \ldots, k\}$, there exists a run $\rho_{j}$ such that $t_{j}$ occurs in the cycle $\theta_{\rho_{j}}$. Let $w_{j}$ be the label of $\rho_{j}$ and $n_{j} \in \mathbb{N}$ such that $\operatorname{dir}\left(\rho_{j}\right) \in(\boldsymbol{c}, \boldsymbol{c})+n_{j}(\boldsymbol{x}, \boldsymbol{y})$. As $\boldsymbol{x} \stackrel{\boldsymbol{c}}{\curvearrowright} \boldsymbol{y}$ there exists a run $\rho$ from $\boldsymbol{c}+\boldsymbol{x}$ to $\boldsymbol{c}+\boldsymbol{y}$ labeled by a word $w$. The cycle $\theta_{\rho}$ shows that $w$ is the label of a cycle on $s_{\gamma}$. Let us consider $n=1+\sum_{j=1}^{k} n_{j}$ and $\sigma=w w_{1} \ldots w_{k}$. Observe that $\sigma$ is the label of a total cycle on $s_{\gamma}$. Hence the Parikh's image of this total cycle provides a Kirchhoff's function $f$ for $\gamma$ such that $\Delta(\sigma)=\Delta(f)$. Observe that $\Delta(\sigma)=n(\boldsymbol{y}-\boldsymbol{x})$. Hence $\boldsymbol{y}-\boldsymbol{x}=\Delta\left(\frac{1}{n} f\right)$ and we have proved that $(\boldsymbol{x}, \boldsymbol{y}) \in R_{\gamma}$.

Lemma 5.15. We have $R_{\gamma} \subseteq \mathbb{Q}_{\geq 0} \stackrel{c}{\curvearrowright}$.

Proof. Lemma 5.7 shows that there exists $\boldsymbol{h}^{\prime} \in \boldsymbol{H}_{\gamma}$ such that $I_{\gamma}=\left\{i \mid \boldsymbol{h}^{\prime}(i)=0\right\}$. From $\boldsymbol{h}^{\prime} \in \boldsymbol{H}_{\gamma}$ we have a run $\rho$ of the form $\boldsymbol{c}+n \boldsymbol{x} \stackrel{w_{1}}{\longrightarrow} \boldsymbol{c}+\boldsymbol{h}^{\prime} \stackrel{w_{2}}{\longrightarrow} \boldsymbol{c}+n \boldsymbol{y}$ for some $n \in \mathbb{N}$ and $w_{1}, w_{2} \in \boldsymbol{A}^{*}$. The cycle $\theta_{\rho}$ shows that there exist cycles $\theta_{1}, \theta_{2}$ on $s_{\gamma}$ labeled by $w_{1}, w_{2}$. We denote by $f_{1}$ and $f_{2}$ the Parikh images of these two cycles. Let $(\boldsymbol{u}, \boldsymbol{v}) \in R_{\gamma}$. By replacing $(\boldsymbol{u}, \boldsymbol{v})$ by a pair in $\mathbb{N}_{>0}(\boldsymbol{u}, \boldsymbol{v})$ we can assume without loss of generality that $\boldsymbol{u}^{\prime}=\boldsymbol{u}-n \boldsymbol{x}$ and $\boldsymbol{v}^{\prime}=\boldsymbol{v}-n \boldsymbol{y}$ are both in $\mathbb{N}^{d}$, and there exists a Kirchhoff's function $f$ such that $f(t) \in \mathbb{N}_{>0}$ and $f(t)>f_{1}(t)+f_{2}(t)$ for every $t \in T_{\gamma}$, and such that $\boldsymbol{v}-\boldsymbol{u}=\Delta(f)$. Since $g=f-\left(f_{1}+f_{2}\right)$ is a Kirchhoff's function satisfying $g(t) \in \mathbb{N}_{>0}$ for every $t \in T_{\gamma}$, Euler's Lemma shows that $g$ is the Parikh's image of a total cycle $\theta$ in $G_{\gamma}$ on $s_{\gamma}$. Let $\sigma$ be the label of this cycle and observe that $\Delta(\sigma)=\Delta(g)=\Delta(f)-\left(\Delta\left(f_{1}\right)+\Delta\left(f_{2}\right)\right)=\boldsymbol{v}-\boldsymbol{u}-\left(\left(\boldsymbol{h}^{\prime}-n \boldsymbol{x}\right)+\left(n \boldsymbol{y}-\boldsymbol{h}^{\prime}\right)\right)=\boldsymbol{v}^{\prime}-\boldsymbol{u}^{\prime}$. Since $\boldsymbol{c}+n \boldsymbol{x} \stackrel{w_{1}}{\longrightarrow} \boldsymbol{c}+\boldsymbol{h}^{\prime} \stackrel{w_{2}}{\longrightarrow} \boldsymbol{c}+n \boldsymbol{y}$ and $n \boldsymbol{x} \leq \boldsymbol{u}, n \boldsymbol{y} \leq \boldsymbol{v}$ we deduce by monotony that for every $m \in \mathbb{N}$ we have:

$$
\boldsymbol{c}+m \boldsymbol{u} \stackrel{w_{1}^{m}}{\longrightarrow} \boldsymbol{c}+m\left(\boldsymbol{h}^{\prime}+\boldsymbol{u}^{\prime}\right) \quad \boldsymbol{c}+m\left(\boldsymbol{h}^{\prime}+\boldsymbol{v}^{\prime}\right) \stackrel{w_{2}^{m}}{\longrightarrow} \boldsymbol{c}+m \boldsymbol{v}
$$

We prove that there exists a run labeled by $\sigma$ from $\boldsymbol{c}+m \boldsymbol{h}^{\prime}$ for some $m \in \mathbb{N}_{>0}$ large enough as follows. We introduce the decomposition of $\sigma$ into $\sigma=\boldsymbol{a}_{1} \ldots \boldsymbol{a}_{k}$ where $\boldsymbol{a}_{j} \in \boldsymbol{A}$. Since $\theta$ is a cycle on the special state $s_{\gamma}$ labeled by $\sigma$, there exists a sequence $\left(s_{j}\right)_{0 \leq j \leq k}$ of states $s_{j} \in S_{\gamma}$ such that $\theta=\left(s_{0}, \boldsymbol{a}_{1}, s_{1}\right) \ldots\left(s_{k-1}, \boldsymbol{a}_{k}, s_{k}\right)$. Let $i \notin I_{\gamma}$ and $j \in\{0, \ldots, k\}$. Since $\boldsymbol{h}^{\prime}(i)>0$ there exists $m_{i, j} \in \mathbb{N}$ such that the $i$ th component of $\boldsymbol{c}+m_{i, j} \boldsymbol{h}^{\prime}+\Delta\left(\boldsymbol{a}_{1} \ldots \boldsymbol{a}_{j}\right)$ is in $\mathbb{N}$. Let $m \in \mathbb{N}_{>0}$ such that $m \geq m_{i, j}$ for every $i \notin I_{\gamma}$ and $j \in\{0, \ldots, k\}$. Note that for every $i \in I_{\gamma}$ and for every $j \in\{0, \ldots, k\}$, the $i$ th component of $\boldsymbol{c}+\Delta\left(\boldsymbol{a}_{1} \ldots \boldsymbol{a}_{j}\right)$ is equal to $s_{j}(i)$ which is in $\mathbb{N}$. We 
have proved that $\boldsymbol{c}+m \boldsymbol{h}^{\prime}+\Delta\left(\boldsymbol{a}_{1} \ldots \boldsymbol{a}_{j}\right) \in \mathbb{N}^{d}$ for every $j \in\{0, \ldots, k\}$. Hence there exists a run from $\boldsymbol{c}+m \boldsymbol{h}^{\prime}$ labeled by $\sigma$.

Let us consider $\ell \in\{0, \ldots, m\}$ and let us introduce $\boldsymbol{z}_{\ell}=(m-\ell) \boldsymbol{u}^{\prime}+\ell \boldsymbol{v}^{\prime}$. Note that $\boldsymbol{z}_{\ell} \in \mathbb{N}^{d}$. By monotony there exists a run from $\boldsymbol{c}+m \boldsymbol{h}^{\prime}+\boldsymbol{z}_{\ell}$ labeled by $\sigma$. Since $\Delta(\sigma)=\boldsymbol{v}^{\prime}-\boldsymbol{u}^{\prime}$, we get $\boldsymbol{z}_{\ell}+\Delta(\sigma)=\boldsymbol{z}_{\ell+1}$. We deduce that $\boldsymbol{c}+m \boldsymbol{h}^{\prime}+\boldsymbol{z}_{\ell} \stackrel{\sigma}{\rightarrow} \boldsymbol{c}+m \boldsymbol{h}^{\prime}+\boldsymbol{z}_{\ell+1}$. Therefore:

$$
\boldsymbol{c}+m\left(\boldsymbol{h}^{\prime}+\boldsymbol{u}^{\prime}\right) \stackrel{\sigma^{m}}{\longrightarrow} \boldsymbol{c}+m\left(\boldsymbol{h}^{\prime}+\boldsymbol{v}^{\prime}\right)
$$

We have proved the lemma by observing that $\boldsymbol{c}+m \boldsymbol{u} \stackrel{w_{1}^{m} \sigma^{m} w_{2}^{m}}{\longrightarrow} \boldsymbol{c}+m \boldsymbol{v}$.

Corollary 5.16. Transformer relations are asymptotically definable periodic relations.

Proof. Lemma 5.14 and Lemma 5.15 show that $\mathbb{Q}_{\geq 0} \stackrel{c}{\curvearrowright}=\bigcup_{\gamma \in \Gamma_{c}} R_{\gamma}$. Since the class $\left\{G_{\gamma} \mid \gamma \in\right.$ $\left.\Gamma_{c}\right\}$ is finite we deduce that the class $\left\{R_{\gamma} \mid \gamma \in \Gamma_{c}\right\}$ is finite. Recall that relations $R_{\gamma}$ are definable in $\mathrm{FO}(\mathbb{Q},+, \leq)$.

\section{Reachability Relations Are Almost Semilinear}

In this section the intersection of the reachability relation $\stackrel{*}{\rightarrow}$ with any Presburger relation $R \subseteq \mathbb{N}^{d} \times \mathbb{N}^{d}$ is proved to be almost semilinear. As a direct corollary we will deduce that post $^{*}(\boldsymbol{X}) \cap \boldsymbol{Y}$ and pre* $(\boldsymbol{Y}) \cap \boldsymbol{X}$ are almost semilinear for every Presburger sets $\boldsymbol{X}, \boldsymbol{Y} \subseteq \mathbb{N}^{d}$. Since Presburger relations are finite unions of linear relations, we can assume that $R=r+P$ where $r \in \mathbb{N}^{d} \times \mathbb{N}^{d}$ and $P \subseteq \mathbb{N}^{d} \times \mathbb{N}^{d}$ is a finitely generated periodic relation. We introduce the set $\Omega$ of runs $\rho$ such that $\operatorname{dir}(\rho) \in R$ equipped with the order $\sqsubseteq$ defined by $\rho \sqsubseteq \rho^{\prime}$ if $\operatorname{dir}\left(\rho^{\prime}\right) \in \operatorname{dir}(\rho)+P$ and $\rho \unlhd \rho^{\prime}$. Since $P$ is finitely generated, Dickson's lemma shows that $\sqsubseteq$ is a well-order. In particular we deduce that the set of minimal runs in $\Omega$ for $\sqsubseteq$, denoted by $\min _{\sqsubseteq}(\Omega)$ is finite.

Lemma 6.1. The intersection of $\stackrel{*}{\rightarrow}$ with $R$ is equal to:

$$
\bigcup_{\rho \in \min _{\sqsubseteq}(\Omega)} \operatorname{dir}(\rho)+(\stackrel{\rho}{\curvearrowright} \cap P)
$$

Proof. Let us first prove that $\operatorname{dir}(\rho)+(\stackrel{\rho}{\curvearrowright} \cap P)$ is included in $\stackrel{*}{\rightarrow} \cap R$ for every run $\rho \in \Omega$. Assume that $\rho=\boldsymbol{c}_{0} \ldots \boldsymbol{c}_{k}$ with $\boldsymbol{c}_{j} \in \mathbb{N}^{d}$ and let $(\boldsymbol{u}, \boldsymbol{v}) \in P$ such that $\boldsymbol{u} \stackrel{\rho}{\curvearrowright} \boldsymbol{v}$. As $\rho \in \Omega$ we deduce that $\left(\boldsymbol{c}_{0}, \boldsymbol{c}_{k}\right) \in R$. As $\boldsymbol{u} \stackrel{\rho}{\curvearrowright} \boldsymbol{v}$ there exists a sequence $\left(\boldsymbol{v}_{j}\right)_{0 \leq j \leq k+1}$ of vectors $\boldsymbol{v}_{j} \in \mathbb{N}^{d}$ such that $\boldsymbol{v}_{0}=\boldsymbol{u}, \boldsymbol{v}_{k+1}=\boldsymbol{v}$ and such that $\boldsymbol{v}_{j} \stackrel{\boldsymbol{c}_{j}}{\curvearrowright} \boldsymbol{v}_{j+1}$ for every $j \in\{0, \ldots, k\}$. In particular there exists a run from $\boldsymbol{c}_{j}+\boldsymbol{v}_{j}$ to $\boldsymbol{c}_{j}+\boldsymbol{v}_{j+1}$ labeled by a word $w_{j} \in \boldsymbol{A}^{*}$. Now just observe that we have a run from $\boldsymbol{c}_{0}+\boldsymbol{v}_{0}$ to $\boldsymbol{c}_{k}+\boldsymbol{v}_{k+1}$ labeled by $w_{0} \boldsymbol{a}_{1} w_{1} \ldots \boldsymbol{a}_{k} w_{k}$ where $\boldsymbol{a}_{j}=\boldsymbol{c}_{j}-\boldsymbol{c}_{j-1}$. Since $\left(\boldsymbol{c}_{0}, \boldsymbol{c}_{k}\right) \in r+P$ and $(\boldsymbol{u}, \boldsymbol{v}) \in P$ we deduce that $\left(\boldsymbol{c}_{0}+\boldsymbol{u}, \boldsymbol{c}_{k}+\boldsymbol{v}\right) \in r+P+P \subseteq R$. Hence $\operatorname{dir}(\rho)+(\boldsymbol{u}, \boldsymbol{v})$ is in $\stackrel{*}{\rightarrow} \cap R$.

Now, let us prove that for every $(\boldsymbol{x}, \boldsymbol{y}) \in R$ such that $\boldsymbol{x} \stackrel{*}{\rightarrow} \boldsymbol{y}$ there exists $\rho \in \min _{\sqsubseteq}(\Omega)$ such that $(\boldsymbol{x}, \boldsymbol{y}) \in \operatorname{dir}(\rho)+(\stackrel{\rho}{\curvearrowright} \cap P)$. There exists a run $\rho^{\prime} \in \Omega$ such that $\operatorname{dir}\left(\rho^{\prime}\right)=(\boldsymbol{x}, \boldsymbol{y})$. Since $\sqsubseteq$ is a well-order, there exists a run $\rho \in \min _{\sqsubseteq}(\Omega)$ such that $\rho \sqsubseteq \rho^{\prime}$. By definition of $\sqsubseteq$ we deduce that $\operatorname{dir}\left(\rho^{\prime}\right) \in \operatorname{dir}(\rho)+(\stackrel{\rho}{\curvearrowright} \cap P)$. 
Since $P$ is finitely generated it is asymptotically definable. From the following lemma we deduce that $\stackrel{\rho}{\curvearrowright} \cap P$ is an asymptotically definable periodic relation. Hence, the previous lemma proved that the intersection of the reachability relation $\stackrel{*}{\rightarrow}$ with every Presburger relation is almost semilinear.

Lemma 6.2. Asymptotically definable periodic sets are stable by intersection.

Proof. If $\boldsymbol{P}_{1}, \boldsymbol{P}_{2} \subseteq \mathbb{Z}^{d}$ are two periodic sets then $\boldsymbol{P}=\boldsymbol{P}_{1} \cap \boldsymbol{P}_{2}$ is a periodic set. Moreover, observe that $\mathbb{Q}_{>0}\left(\boldsymbol{P}_{1} \cap \boldsymbol{P}_{2}\right)=\left(\mathbb{Q}_{>0} \boldsymbol{P}_{1}\right) \cap\left(\mathbb{Q}_{>0} \boldsymbol{P}_{2}\right)$. Hence, if $\boldsymbol{P}_{1}, \boldsymbol{P}_{2}$ are asymptotically definable then $\boldsymbol{P}$ is also asymptotically definable.

We deduce the following corollary.

Corollary 6.3. The sets $\operatorname{post}^{*}(\boldsymbol{X}) \cap \boldsymbol{Y}$ and $\operatorname{pre}^{*}(\boldsymbol{Y}) \cap \boldsymbol{X}$ are almost semilinear for every Presburger sets $\boldsymbol{X}, \boldsymbol{Y} \subseteq \mathbb{N}^{d}$.

Proof. Let us consider the Presburger relation $R=\boldsymbol{X} \times \boldsymbol{Y}$ and observe that $\operatorname{post}^{*}(\boldsymbol{X}) \cap \boldsymbol{Y}=$ $f(\stackrel{*}{\rightarrow} \cap R)$ and $\operatorname{pre}^{*}(\boldsymbol{Y}) \cap \boldsymbol{X}=g(\stackrel{*}{\rightarrow} \cap R)$ where $f, g: \mathbb{Q}^{d} \times \mathbb{Q}^{d} \rightarrow \mathbb{Q}^{d}$ and defined by $f(\boldsymbol{x}, \boldsymbol{y})=\boldsymbol{y}$ and $g(\boldsymbol{x}, \boldsymbol{y})=\boldsymbol{x}$. Now just observe that for every $r \in \mathbb{N}^{d} \times \mathbb{N}^{d}$, for every asymptotically definable periodic relation $P \subseteq \mathbb{N}^{d} \times \mathbb{N}^{d}$, and for every $h \in\{f, g\}$ we have $h(r+P)=h(r)+h(P)$. Moreover $h(P)$ is a periodic set and the conic set $\mathbb{Q}_{\geq 0} h(P)$ is equal to $h(\mathbb{Q} \geq 0)$ which is definable in $\mathrm{FO}(\mathbb{Q},+, \leq)$.

\section{Dimension}

In this section we introduce a dimension function for the subsets of $\mathbb{Z}^{d}$ and we characterize the dimension of periodic sets.

A vector space is a set $\boldsymbol{V} \subseteq \mathbb{Q}^{d}$ such that $\mathbf{0} \in \boldsymbol{V}, \boldsymbol{V}+\boldsymbol{V} \subseteq \boldsymbol{V}$ and such that $\mathbb{Q} \boldsymbol{V} \subseteq \boldsymbol{V}$. Let $\boldsymbol{X} \subseteq \mathbb{Q}^{d}$. The following set $\boldsymbol{V}$ is a vector space called the vector space generated by $\bar{X}$.

$$
\boldsymbol{V}=\left\{\sum_{j=1}^{k} \lambda_{j} \boldsymbol{x}_{j} \mid k \in \mathbb{N} \text { and }\left(\lambda_{j}, \boldsymbol{x}_{j}\right) \in \mathbb{Q} \times \boldsymbol{X}\right\}
$$

This vector space is the minimal for the inclusion among the vector spaces that contain $\boldsymbol{X}$. Let us recall that every vector space $\boldsymbol{V}$ is generated by a finite set. The $\operatorname{rank} \operatorname{rank}(\boldsymbol{V})$ of a vector space $\boldsymbol{V}$ is the minimal natural number $m \in \mathbb{N}$ such that there exists a finite set $\boldsymbol{X}$ with $m$ vectors that generates $\boldsymbol{V}$. Let us recall that $\operatorname{rank}(\boldsymbol{V}) \leq d$ for every vector space $\boldsymbol{V} \subseteq \mathbb{Q}^{d}$ and $\operatorname{rank}(\boldsymbol{V}) \leq \operatorname{rank}(\boldsymbol{W})$ for every pair of vector spaces $\boldsymbol{V} \subseteq \boldsymbol{W}$. Moreover, if $\boldsymbol{V}$ is strictly included in $\boldsymbol{W}$ then $\operatorname{rank}(\boldsymbol{V})<\operatorname{rank}(\boldsymbol{W})$.

Example 7.1. Vector spaces $\boldsymbol{V}$ included in $\mathbb{Q}^{2}$ satisfy $\operatorname{rank}(\boldsymbol{V}) \in\{0,1,2\}$. Moreover these vectors spaces can be classified as follows : $\operatorname{rank}(\boldsymbol{V})=0$ if and only if $\boldsymbol{V}=\{\mathbf{0}\}, \operatorname{rank}(\boldsymbol{V})=1$ if and only if $\boldsymbol{V}=\mathbb{Q} \boldsymbol{v}$ with $\boldsymbol{v} \in \mathbb{Q}^{2} \backslash\{\mathbf{0}\}$, and $\operatorname{rank}(\boldsymbol{V})=2$ if and only if $\boldsymbol{V}=\mathbb{Q}^{2}$.

The dimension of a set $\boldsymbol{X} \subseteq \mathbb{Z}^{d}$ is the minimal integer $m \in\{-1, \ldots, d\}$ such that $\boldsymbol{X} \subseteq$ $\bigcup_{j=1}^{k} \boldsymbol{b}_{j}+\boldsymbol{V}_{j}$ where $\boldsymbol{b}_{j} \in \mathbb{Z}^{d}$ and $\boldsymbol{V}_{j} \subseteq \mathbb{Q}^{d}$ is a vector space satisfying $\operatorname{rank}\left(\boldsymbol{V}_{j}\right) \leq m$ for every $j$. We denote by $\operatorname{dim}(\boldsymbol{X})$ the dimension of $\boldsymbol{X}$. Observe that $\operatorname{dim}(\boldsymbol{v}+\boldsymbol{X})=\operatorname{dim}(\boldsymbol{X})$ for every $\boldsymbol{X} \subseteq \mathbb{Z}^{d}$ and for every $\boldsymbol{v} \in \mathbb{Z}^{d}$. Moreover we have $\operatorname{dim}(\boldsymbol{X})=-1$ if and only if $\boldsymbol{X}$ is empty. Note that $\operatorname{dim}(\boldsymbol{X} \cup \boldsymbol{Y})=\max \{\operatorname{dim}(\boldsymbol{X}), \operatorname{dim}(\boldsymbol{Y})\}$ for every subsets $\boldsymbol{X}, \boldsymbol{Y} \subseteq \mathbb{Z}^{d}$. 
Example 7.2. Let $\boldsymbol{X}=\{-10, \ldots, 10\} \times \mathbb{Z}$. Observe that $\operatorname{dim}(\boldsymbol{X}) \leq 1$ since the set $\boldsymbol{X}$ is included in $\bigcup_{\boldsymbol{b} \in\{-10, \ldots, 10\} \times\{0\}} \boldsymbol{b}+\boldsymbol{V}$ where $\boldsymbol{V}=\{0\} \times \mathbb{Q}$.

Lemma 7.3. Let $\boldsymbol{P} \subseteq \mathbb{Z}^{d}$ be a periodic set included in $\bigcup_{j=1}^{k} \boldsymbol{b}_{j}+\boldsymbol{V}_{j}$ where $k \in \mathbb{N}_{>0}, \boldsymbol{b}_{j} \in \mathbb{Z}^{d}$ and $\boldsymbol{V}_{j} \subseteq \mathbb{Q}^{d}$ is a vector space. There exists $j \in\{1, \ldots, k\}$ such that $\boldsymbol{P} \subseteq \boldsymbol{V}_{j}$ and $\boldsymbol{b}_{j} \in \boldsymbol{V}_{j}$.

Proof. Let us first prove by induction over $k \in \mathbb{N}_{>0}$ that for every periodic set $\boldsymbol{P} \subseteq \mathbb{Z}^{d}$ included in $\bigcup_{j=1}^{k} \boldsymbol{V}_{j}$ where $\boldsymbol{V}_{j} \subseteq \mathbb{Q}^{d}$ is a vector space, there exists $j \in\{1, \ldots, k\}$ such that $\boldsymbol{P} \subseteq \boldsymbol{V}_{j}$. The rank $k=1$ is immediate. Assume the rank $k$ proved and let us prove the rank $k+1$. Let $\boldsymbol{P}$ be a periodic set included in $\bigcup_{j=1}^{k+1} \boldsymbol{V}_{j}$ where $\boldsymbol{V}_{j} \subseteq \mathbb{Q}^{d}$ is a vector space. If $\boldsymbol{P} \subseteq \boldsymbol{V}_{k+1}$ the induction is proved. So we can assume that there exists $\boldsymbol{p} \in \boldsymbol{P} \backslash \boldsymbol{V}_{k+1}$. Let $\boldsymbol{x} \in \boldsymbol{P}$. Since $\boldsymbol{p}+n \boldsymbol{x} \in \boldsymbol{P}$ for every $n \in \mathbb{N}$, the pigeon-hole principle shows that there exist $j \in\{1, \ldots, k+1\}$ and $n<m$ such that $n \boldsymbol{p}+\boldsymbol{x}$ and $m \boldsymbol{p}+\boldsymbol{x}$ are both in $\boldsymbol{V}_{j}$. In particular the difference of this two vectors is in $\boldsymbol{V}_{j}$. Since this difference is $(m-n) \boldsymbol{p}$ and $\boldsymbol{p} \notin \boldsymbol{V}_{k+1}$ we get $j \in\{1, \ldots, k\}$. Observe that $n(m \boldsymbol{p}+\boldsymbol{x})-m(n \boldsymbol{p}+\boldsymbol{x})$ is the difference of two vectors in $\boldsymbol{V}_{j}$. Thus this vector is in $\boldsymbol{V}_{j}$ and we deduce that $\boldsymbol{x} \in \boldsymbol{V}_{j}$. We have shown that $\boldsymbol{P} \subseteq \bigcup_{j=1}^{k} \boldsymbol{V}_{j}$. By induction there exists $j \in\{1, \ldots, k\}$ such that $\boldsymbol{P} \subseteq \boldsymbol{V}_{j}$. We have proved the induction.

Finally, assume that $\boldsymbol{P} \subseteq \mathbb{Z}^{d}$ is a periodic set included in $\bigcup_{j=1}^{k} \boldsymbol{b}_{j}+\boldsymbol{V}_{j}$ where $k \in \mathbb{N}_{>0}$, $\boldsymbol{b}_{j} \in \mathbb{Z}^{d}$ and $\boldsymbol{V}_{j} \subseteq \mathbb{Q}^{d}$ is a vector space. Let $J$ be the set of $j \in\{1, \ldots, k\}$ such that $\boldsymbol{b}_{j} \in \boldsymbol{V}_{j}$ and let us prove that $\boldsymbol{P} \subseteq \bigcup_{j \in J} \boldsymbol{V}_{j}$. Let $\boldsymbol{p} \in \boldsymbol{P}$. Since $n \boldsymbol{p} \in \boldsymbol{P}$ for every $n \in \mathbb{N}$, there exist $j \in\{1, \ldots, k\}$ and $n<m$ such that $n \boldsymbol{p}$ and $m \boldsymbol{p}$ are both in $\boldsymbol{b}_{j}+\boldsymbol{V}_{j}$. The difference of these two vectors shows that $(m-n) \boldsymbol{p}$ is in $\boldsymbol{V}_{j}$. From $\boldsymbol{b}_{j} \in n \boldsymbol{p}-\boldsymbol{V}_{j} \subseteq \boldsymbol{V}_{j}$ we deduce that $j \in J$. Thus $\boldsymbol{P} \subseteq \bigcup_{j \in J} \boldsymbol{V}_{j}$. As $\mathbf{0} \in \boldsymbol{P}$ we deduce that $J \neq \emptyset$ and from the previous paragraph, there exists $j \in J$ such that $\boldsymbol{P} \subseteq \boldsymbol{V}_{j}$.

Lemma 7.4. We have $\operatorname{dim}(\boldsymbol{P})=\operatorname{rank}(\boldsymbol{V})$ for every periodic set $\boldsymbol{P}$ where $\boldsymbol{V}$ is the vector space generated by $\boldsymbol{P}$.

Proof. Since $\boldsymbol{P} \subseteq \boldsymbol{V}$ we deduce that $\operatorname{dim}(\boldsymbol{P}) \leq \operatorname{rank}(\boldsymbol{V})$. For the converse inequality, there exist $k \in \mathbb{N},\left(\boldsymbol{b}_{j}\right)_{1 \leq j \leq k}$ a sequence of vectors $\boldsymbol{b}_{j} \in \mathbb{Z}^{d}$ and a sequence $\left(\boldsymbol{V}_{j}\right)_{1 \leq j \leq k}$ of vector spaces $\boldsymbol{V}_{j} \subseteq \mathbb{Q}^{d}$ such that $\boldsymbol{P} \subseteq \bigcup_{j=1}^{k} \boldsymbol{b}_{j}+\boldsymbol{V}_{j}$ and such that $\operatorname{rank}\left(\boldsymbol{V}_{j}\right) \leq \operatorname{dim}(\boldsymbol{P})$ for every $j$. Since $\boldsymbol{P}$ is non empty we deduce that $k \in \mathbb{N}_{>0}$. Lemma 7.3 proves that there exists $j \in\{1, \ldots, k\}$ such that $\boldsymbol{P} \subseteq \boldsymbol{V}_{j}$ and $\boldsymbol{b}_{j} \in \boldsymbol{V}_{j}$. By minimality of the vector space generated by $\boldsymbol{P}$ we get $\boldsymbol{V} \subseteq \boldsymbol{V}_{j}$. Hence $\operatorname{rank}(\boldsymbol{V}) \leq \operatorname{rank}\left(\boldsymbol{V}_{j}\right)$. From $\operatorname{rank}\left(\boldsymbol{V}_{j}\right) \leq \operatorname{dim}(\boldsymbol{P})$ we get $\operatorname{rank}(\boldsymbol{V}) \leq \operatorname{dim}(\boldsymbol{P})$.

\section{Linearizations}

A linearization of an almost semilinear set $\boldsymbol{X}$ is a set $\bigcup_{j=1}^{k} \boldsymbol{b}_{j}+\left(\boldsymbol{P}_{j}-\boldsymbol{P}_{j}\right) \cap \mathbb{Q}_{\geq 0} \boldsymbol{P}_{j}$ where $\boldsymbol{b}_{j} \in \mathbb{Z}^{d}$ and $\boldsymbol{P}_{j} \subseteq \mathbb{Z}^{d}$ is an asymptotically definable periodic set such that $\boldsymbol{X}=\bigcup_{j=1}^{k} \boldsymbol{b}_{j}+\boldsymbol{P}_{j}$. Let us recall that every subgroup of $\left(\mathbb{Z}^{d},+\right)$ is finitely generated[14]. Moreover, since $\mathrm{FO}(\mathbb{Q},+, \leq, 0)$ admits a quantifier elimination algorithm, we deduce that linearizations are definable in the Presburger arithmetic.

Remark 8.1. Almost semilinear sets can have multiple linearizations.

In this section we show that if $\boldsymbol{X}, \boldsymbol{Y} \subseteq \mathbb{Z}^{d}$ are two non-empty almost semilinear sets with an empty intersection then every linearizations $\boldsymbol{S}, \boldsymbol{T}$ of $\boldsymbol{X}, \boldsymbol{Y}$ satisfy:

$$
\operatorname{dim}(\boldsymbol{S} \cap \boldsymbol{T})<\operatorname{dim}(\boldsymbol{X} \cup \boldsymbol{Y})
$$




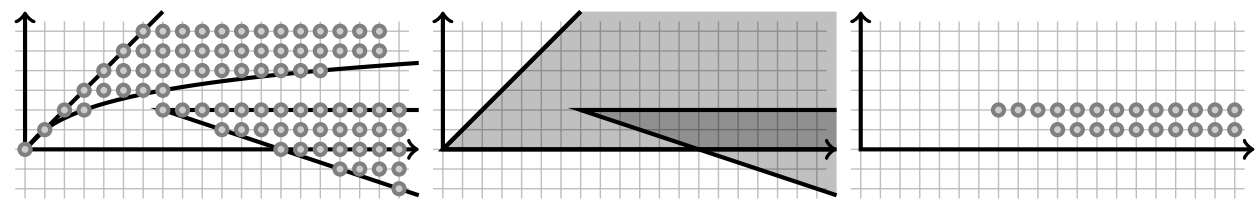

Figure 1: From left to right: sets $\boldsymbol{X}$ and $\boldsymbol{Y}$, sets $\boldsymbol{u}+\mathbb{Q}_{\geq 0} \boldsymbol{P}$ and $\boldsymbol{v}+\mathbb{Q}_{\geq 0} \boldsymbol{Q}$, and set $\boldsymbol{S} \cap \boldsymbol{T}$.

Example 8.2. Sets introduced in this example are depicted in Figure 1. Let us introduce the asymptotically definable periodic set $\boldsymbol{P}=\left\{\boldsymbol{p} \in \mathbb{N}^{2} \mid \boldsymbol{p}(2) \leq \boldsymbol{p}(1) \leq 2^{\boldsymbol{p}(2)}-1\right\}$ and the finitely generated periodic set $\boldsymbol{Q}=\mathbb{N}(1,0)+\mathbb{N}(3,-1)$. We introduce the almost semilinear sets $\boldsymbol{X}=\boldsymbol{u}+\boldsymbol{P}$ and $\boldsymbol{Y}=\boldsymbol{v}+\boldsymbol{Q}$ where $\boldsymbol{u}=(0,0)$ and $\boldsymbol{v}=(7,2)$. Observe that $\boldsymbol{X} \cap \boldsymbol{Y}$ is empty and $\operatorname{dim}(\boldsymbol{X} \cup \boldsymbol{Y})=2$. Let us consider linearizations $\boldsymbol{S}, \boldsymbol{T}$ of $\boldsymbol{X}, \boldsymbol{Y}$ defined by $\boldsymbol{S}=\boldsymbol{u}+\boldsymbol{P}^{\prime}$ and $\boldsymbol{T}=\boldsymbol{v}+\boldsymbol{Q}^{\prime}$ where $\boldsymbol{P}^{\prime}=(\boldsymbol{P}-\boldsymbol{P}) \cap \mathbb{Q}_{\geq 0} \boldsymbol{P}$ and $\boldsymbol{Q}^{\prime}=(\boldsymbol{Q}-\boldsymbol{Q}) \cap \mathbb{Q}_{\geq 0} \boldsymbol{Q}$. Observe that $\boldsymbol{P}^{\prime}=\{(0,0)\} \cup\left\{\boldsymbol{p} \in \mathbb{N}_{>0}^{2} \mid \boldsymbol{p}(2) \leq \boldsymbol{p}(1)\right\}$ and $\boldsymbol{Q}^{\prime}=\boldsymbol{Q}$. Note that the intersection $\boldsymbol{S} \cap \boldsymbol{T}$ is non empty since it is equal to $\{(7,2),(10,1)\}+\mathbb{N}(1,0)$. In particular $\operatorname{dim}(\boldsymbol{S} \cap \boldsymbol{T}) \leq 1$ and we get $\operatorname{dim}(\boldsymbol{S} \cap \boldsymbol{T})<\operatorname{dim}(\boldsymbol{X} \cup \boldsymbol{Y})$.

Lemma 8.3. Assume that $\boldsymbol{b}+\boldsymbol{M} \subseteq(\boldsymbol{P}-\boldsymbol{P}) \cap \mathbb{Q}_{\geq 0} \boldsymbol{P}$ where $\boldsymbol{b} \in \mathbb{Z}^{d}$ and $\boldsymbol{M}, \boldsymbol{P} \subseteq \mathbb{Z}^{d}$ are two periodic sets. Let $\boldsymbol{a}$ be a vector of the form $\boldsymbol{m}_{1}+\cdots+\boldsymbol{m}_{k}$ where $\left(\boldsymbol{m}_{j}\right)_{1 \leq j \leq k}$ is a sequence of vectors $\boldsymbol{m}_{j} \in \boldsymbol{M}$ that generates a vector space that contains $\boldsymbol{P}$. There exists $k \in \mathbb{N}_{>0}$ such that $\boldsymbol{b}+k \mathbb{N}_{>0} \boldsymbol{a} \subseteq \boldsymbol{P}$.

Proof. Since $\boldsymbol{b} \in \boldsymbol{P}-\boldsymbol{P}$ there exists $\boldsymbol{p}_{+}, \boldsymbol{p}_{-} \in \boldsymbol{P}$ such that $\boldsymbol{b}=\boldsymbol{p}_{+}-\boldsymbol{p}_{-}$. As the sequence $\left(\boldsymbol{m}_{j}\right)_{1 \leq j \leq k}$ generates a vector space that contains $\boldsymbol{P}$, we get $\boldsymbol{p}_{+} \in \sum_{j=1}^{k} \mathbb{Q} \boldsymbol{m}_{j}$. Hence there exists $z \in \mathbb{N}_{>0}$ such that $-z \boldsymbol{p}_{+} \in \sum_{j=1}^{k} \mathbb{Z} \boldsymbol{m}_{j}$. By definition of $\boldsymbol{a}$, there exists $n \in \mathbb{N}_{>0}$ such that $-z \boldsymbol{p}_{+}+n \boldsymbol{a} \in \sum_{j=1}^{k} \mathbb{N} \boldsymbol{m}_{j}$. Hence $\boldsymbol{b}-z \boldsymbol{p}_{+}+n \boldsymbol{a} \in \boldsymbol{b}+\sum_{j=1}^{k} \mathbb{N} \boldsymbol{m}_{j}$. Since this set is included in $\mathbb{Q}_{\geq 0} \boldsymbol{P}$ and $(z-1) \boldsymbol{p}_{+} \in \boldsymbol{P}$ we deduce that $\left(\boldsymbol{b}-z \boldsymbol{p}_{+}+n \boldsymbol{a}\right)+(z-1) \boldsymbol{p}_{+}$is in $\mathbb{Q}_{\geq 0} \boldsymbol{P}$. Note that this vector is equal to $-\boldsymbol{p}_{-}+n \boldsymbol{a}$ since $\boldsymbol{b}=\boldsymbol{p}_{+}-\boldsymbol{p}_{-}$. Hence, there exists $s \in \mathbb{N}_{>0}$ such that $s\left(-\boldsymbol{p}_{-}+n \boldsymbol{a}\right) \in \boldsymbol{P}$. Let $k=s n$ and observe that $-\boldsymbol{p}_{-}+k \boldsymbol{a}=s\left(-\boldsymbol{p}_{-}+n \boldsymbol{a}\right)+(s-1) \boldsymbol{p}_{-}$. Hence $-\boldsymbol{p}_{-}+k \boldsymbol{a} \in \boldsymbol{P}$. Since $\boldsymbol{b}+k \boldsymbol{a}=\left(-\boldsymbol{p}_{-}+k \boldsymbol{a}\right)+\boldsymbol{p}_{+}$and $k \boldsymbol{a}=\left(-\boldsymbol{p}_{-}+k \boldsymbol{a}\right)+\boldsymbol{p}_{-}$we deduce that $\boldsymbol{b}+k \boldsymbol{a}$ and $k \boldsymbol{a}$ are both in $\boldsymbol{P}$. In particular $\boldsymbol{b}+k \mathbb{N}_{>0} \boldsymbol{a} \subseteq \boldsymbol{P}$.

Corollary 8.4. Let $\boldsymbol{X}, \boldsymbol{Y} \subseteq \mathbb{Z}^{d}$ be two non-empty almost semilinear sets with an empty intersection. For every linearizations $\boldsymbol{S}, \boldsymbol{T}$ of $\boldsymbol{X}, \boldsymbol{Y}$ we have:

$$
\operatorname{dim}(\boldsymbol{S} \cap \boldsymbol{T})<\operatorname{dim}(\boldsymbol{X} \cup \boldsymbol{Y})
$$

Proof. We can assume that $\boldsymbol{X}=\boldsymbol{u}+\boldsymbol{P}, \boldsymbol{Y}=\boldsymbol{v}+\boldsymbol{Q}$ where $\boldsymbol{u}, \boldsymbol{v} \in \mathbb{Z}^{d}$ and $\boldsymbol{P}, \boldsymbol{Q} \subseteq \mathbb{Z}^{d}$ are two asymptotically definable periodic sets such that $\boldsymbol{X} \cap \boldsymbol{Y}=\emptyset$ and we can assume that $\boldsymbol{S}=\boldsymbol{u}+\boldsymbol{P}^{\prime}$ where $\boldsymbol{P}^{\prime}=(\boldsymbol{P}-\boldsymbol{P}) \cap \mathbb{Q}_{\geq 0} \boldsymbol{P}$ and $\boldsymbol{T}=\boldsymbol{v}+\boldsymbol{Q}^{\prime}$ where $\boldsymbol{Q}^{\prime}=(\boldsymbol{Q}-\boldsymbol{Q}) \cap \mathbb{Q}_{\geq 0} \boldsymbol{Q}$. Let $\boldsymbol{U}$ and $\boldsymbol{V}$ be the vector spaces generated by $\boldsymbol{P}$ and $\boldsymbol{Q}$. Lemma 7.4 shows that $\operatorname{dim}(\boldsymbol{X})=\operatorname{rank}(\boldsymbol{U})$ and $\operatorname{dim}(\boldsymbol{Y})=\operatorname{rank}(\boldsymbol{V})$. Note that $\boldsymbol{S} \cap \boldsymbol{T}$ is a Presburger set and in particular a finite union of linear sets. If this set is empty the corollary is proved. Otherwise there exists $\boldsymbol{b} \in \mathbb{Z}^{d}$ and a finitely generated periodic set $\boldsymbol{M} \subseteq \mathbb{Z}^{d}$ such that $\boldsymbol{b}+\boldsymbol{M} \subseteq \boldsymbol{S} \cap \boldsymbol{T}$ and such that $\operatorname{dim}(\boldsymbol{S} \cap \boldsymbol{T})=\operatorname{dim}(\boldsymbol{b}+\boldsymbol{M})$. Let $\boldsymbol{W}$ be the vector space generated by $\boldsymbol{M}$. Observe that $\boldsymbol{b}+\boldsymbol{M} \subseteq(\boldsymbol{u}+\boldsymbol{U}) \cap(\boldsymbol{v}+\boldsymbol{V})$. Hence for every $\boldsymbol{m} \in \boldsymbol{M}$ since $\boldsymbol{b}+\boldsymbol{m}-\boldsymbol{u}$ and $\boldsymbol{b}+2 \boldsymbol{m}-\boldsymbol{u}$ are both in $\boldsymbol{U}$ the difference is also in $\boldsymbol{U}$. Hence $\boldsymbol{m} \in \boldsymbol{U}$. We deduce that $\boldsymbol{M} \subseteq \boldsymbol{U}$ and symmetrically 
$\boldsymbol{M} \subseteq \boldsymbol{V}$. As $\boldsymbol{M}$ is included in the vector space $\boldsymbol{U} \cap \boldsymbol{V}$, by minimality of $\boldsymbol{W}$, we get $\boldsymbol{W} \subseteq \boldsymbol{U} \cap \boldsymbol{V}$. Assume by contradiction that $\boldsymbol{W}=\boldsymbol{U}$ and $\boldsymbol{W}=\boldsymbol{V}$. Since $\boldsymbol{M}$ is finitely generated, there exists a sequence $\left(\boldsymbol{m}_{j}\right)_{1 \leq j \leq k}$ of vectors $\boldsymbol{m}_{j} \in \boldsymbol{M}$ such that $\boldsymbol{M}=\mathbb{N} \boldsymbol{m}_{1}+\cdots+\mathbb{N} \boldsymbol{m}_{k}$. Let $\boldsymbol{a}=\boldsymbol{m}_{1}+\cdots+\boldsymbol{m}_{k}$. From $\boldsymbol{b}-\boldsymbol{u}+\boldsymbol{M} \subseteq(\boldsymbol{P}-\boldsymbol{P}) \cap \mathbb{Q}_{\geq 0} \boldsymbol{P}$ and Lemma 8.3 we deduce that there exists $k \in \mathbb{N}_{>0}$ such that $\boldsymbol{b}-\boldsymbol{u}+k \mathbb{N}_{>0} \boldsymbol{a} \subseteq \boldsymbol{P}$. From $\boldsymbol{b}-\boldsymbol{v}+\boldsymbol{M} \subseteq(\boldsymbol{Q}-\boldsymbol{Q}) \cap \mathbb{Q}_{\geq 0} \boldsymbol{Q}$ and Lemma 8.3 we deduce that there exists $k^{\prime} \in \mathbb{N}_{>0}$ such that $\boldsymbol{b}-\boldsymbol{v}+k^{\prime} \mathbb{N}_{>0} \boldsymbol{a} \subseteq \boldsymbol{Q}$. In particular $\boldsymbol{b}+k k^{\prime} \boldsymbol{a} \in(\boldsymbol{u}+\boldsymbol{P}) \cap(\boldsymbol{v}+\boldsymbol{Q})$ and we get a contradiction since this intersection is empty. Thus $\boldsymbol{W} \neq \boldsymbol{U}$ or $\boldsymbol{W} \neq \boldsymbol{V}$. Since $\boldsymbol{W} \subseteq \boldsymbol{U} \cap \boldsymbol{V}$ we deduce that $\boldsymbol{W}$ is strictly included in $\boldsymbol{U}$ or in $\boldsymbol{V}$. Hence $\operatorname{rank}(\boldsymbol{W})<\max \{\operatorname{rank}(\boldsymbol{U}), \operatorname{rank}(\boldsymbol{V})\}=\operatorname{dim}(\boldsymbol{X} \cup \boldsymbol{Y})$. From Lemma 7.4 we get $\operatorname{dim}(\boldsymbol{M})=\operatorname{rank}(\boldsymbol{W})$ and since $\operatorname{dim}(\boldsymbol{M})=\operatorname{dim}(\boldsymbol{S} \cap \boldsymbol{T})$ the corollary is proved.

\section{$9 \quad$ Presburger Invariants}

We introduce the notion of separators. A separator is a pair $(\boldsymbol{X}, \boldsymbol{Y})$ of Presburger sets $\boldsymbol{X}, \boldsymbol{Y} \subseteq$ $\mathbb{N}^{d}$ such that there does not exist a run from a configuration in $\boldsymbol{X}$ to a configuration in $\boldsymbol{Y}$. In particular $\boldsymbol{X} \cap \boldsymbol{Y}=\emptyset$. The Presburger set $\boldsymbol{D}=\mathbb{N}^{d} \backslash(\boldsymbol{X} \cup \boldsymbol{Y})$ is called the domain of $(\boldsymbol{X}, \boldsymbol{Y})$. We observe that a separator $(\boldsymbol{X}, \boldsymbol{Y})$ with an empty domain is a partition of $\mathbb{N}^{d}$ such that $\boldsymbol{X}$ is a Presburger forward inductive invariant and $\boldsymbol{Y}$ is a Presburger backward inductive invariant.

Lemma 9.1. Let $\left(\boldsymbol{X}_{0}, \boldsymbol{Y}_{0}\right)$ be a separator with a non-empty domain $\boldsymbol{D}_{0}$. There exists a separator $(\boldsymbol{X}, \boldsymbol{Y})$ with a domain $\boldsymbol{D}$ such that $\boldsymbol{X}_{0} \subseteq \boldsymbol{X}, \boldsymbol{Y}_{0} \subseteq \boldsymbol{Y}$ and $\operatorname{dim}(\boldsymbol{D})<\operatorname{dim}\left(\boldsymbol{D}_{0}\right)$.

Proof. As $\boldsymbol{X}_{0}, \boldsymbol{D}_{0}$ are Presburger sets, Corollary 6.3 shows that $\boldsymbol{H}=\operatorname{post}^{*}\left(\boldsymbol{X}_{0}\right) \cap \boldsymbol{D}_{0}$ is an almost semilinear set. We introduce a linearization $\boldsymbol{S}$ of this set. Since $\left(\boldsymbol{X}_{0}, \boldsymbol{Y}_{0}\right)$ is a separator, the intersection post* $\left(\boldsymbol{X}_{0}\right) \cap \boldsymbol{Y}_{0}$ is empty. Moreover, as post* $\left(\boldsymbol{X}_{0}\right) \cap \boldsymbol{D}_{0} \subseteq \boldsymbol{S}$, we deduce that the set $\boldsymbol{Y}=\boldsymbol{Y}_{0} \cup\left(\boldsymbol{D}_{0} \backslash \boldsymbol{S}\right)$ is such that $\operatorname{post}^{*}\left(\boldsymbol{X}_{0}\right) \cap \boldsymbol{Y}=\emptyset$. Hence $\left(\boldsymbol{X}_{0}, \boldsymbol{Y}\right)$ is a separator. Symmetrically, as $\boldsymbol{D}_{0}, \boldsymbol{Y}$ are Presburger sets, Corollary 6.3 shows that $\boldsymbol{K}=\operatorname{pre}^{*}(\boldsymbol{Y}) \cap \boldsymbol{D}_{0}$ is an almost semilinear set. We introduce a linearization $\boldsymbol{T}$ of this set. Since $\left(\boldsymbol{X}_{0}, \boldsymbol{Y}\right)$ is a separator, the intersection $\operatorname{pre}^{*}(\boldsymbol{Y}) \cap \boldsymbol{X}_{0}$ is empty. Moreover, as $\operatorname{pre}^{*}(\boldsymbol{Y}) \cap \boldsymbol{D}_{0} \subseteq \boldsymbol{T}$, we deduce that the set $\boldsymbol{X}=\boldsymbol{X}_{0} \cup\left(\boldsymbol{D}_{0} \backslash \boldsymbol{T}\right)$ is such that $\operatorname{pre}^{*}(\boldsymbol{Y}) \cap \boldsymbol{X}=\emptyset$. Hence $(\boldsymbol{X}, \boldsymbol{Y})$ is a separator.

Let us introduce the domain $\boldsymbol{D}$ of $(\boldsymbol{X}, \boldsymbol{Y})$ and observe that $\boldsymbol{D}=\boldsymbol{D}_{0} \cap \boldsymbol{S} \cap \boldsymbol{T}$. If $\boldsymbol{H}$ or $\boldsymbol{K}$ is empty then $\boldsymbol{S}$ or $\boldsymbol{T}$ is empty and in particular $\boldsymbol{D}$ is empty and the lemma is proved. So we can assume that $\boldsymbol{H}$ and $\boldsymbol{K}$ are non empty. Since $\boldsymbol{H} \subseteq \operatorname{post}^{*}\left(\boldsymbol{X}_{0}\right) \subseteq \operatorname{post}^{*}(\boldsymbol{X})$ and $\boldsymbol{K} \subseteq \operatorname{pre}^{*}(\boldsymbol{Y})$ and $(\boldsymbol{X}, \boldsymbol{Y})$ is a separator, we deduce that $\boldsymbol{H} \cap \boldsymbol{K}=\emptyset$. Moreover as $\boldsymbol{H}, \boldsymbol{K} \subseteq \boldsymbol{D}_{0}$ we deduce that $\operatorname{dim}(\boldsymbol{H} \cup \boldsymbol{K}) \leq \operatorname{dim}\left(\boldsymbol{D}_{0}\right)$. As $\boldsymbol{S}$ and $\boldsymbol{T}$ are linearizations of the non-empty almost semilinear sets $\boldsymbol{H}, \boldsymbol{K}$ and $\boldsymbol{H} \cap \boldsymbol{K}=\emptyset$, Corollary 8.4 shows that $\operatorname{dim}(\boldsymbol{S} \cap \boldsymbol{T})<\operatorname{dim}(\boldsymbol{H} \cup \boldsymbol{K})$. Therefore $\operatorname{dim}(\boldsymbol{D})<\operatorname{dim}\left(\boldsymbol{D}_{0}\right)$.

We deduce the main theorem of this paper.

Theorem 9.2. For every $\boldsymbol{x}, \boldsymbol{y} \in \mathbb{N}^{d}$ such that there does not exist a run from $\boldsymbol{x}$ to $\boldsymbol{y}$, then there exists a pair $(\boldsymbol{X}, \boldsymbol{Y})$ of disjoint Presburger sets $\boldsymbol{X}, \boldsymbol{Y} \subseteq \mathbb{N}^{d}$ such that $\boldsymbol{X}$ is a forward inductive invariant that contains $\boldsymbol{x}$ and $\boldsymbol{Y}$ is a backward inductive invariant that contains $\boldsymbol{y}$.

Proof. Observe that $(\{\boldsymbol{x}\},\{\boldsymbol{y}\})$ is a separator.Thanks to Lemma 9.1 with an immediate induction over the dimension of the domains we deduce that there exists a separator $(\boldsymbol{X}, \boldsymbol{Y})$ with an empty domain such that $\boldsymbol{x} \in \boldsymbol{X}$ and $\boldsymbol{y} \in \boldsymbol{Y}$. 


\section{Conclusion}

The reachability problem for vector addition systems can be solved with a simple algorithm based on inductive invariants definable in the Presburger arithmetic. This algorithm does not require the classical KLMST decomposition. Note however that the complexity of this algorithm is still open. In fact, the complexity depends on the minimal length of a run from $\boldsymbol{x}$ to $\boldsymbol{y}$ when such a run exists, or the minimal length of a Presburger formula denoting a forward inductive invariant $\boldsymbol{X}$ such that $\boldsymbol{x} \in \boldsymbol{X}$ and $\boldsymbol{y} \notin \boldsymbol{X}$ when such a formula exists. We left as an open question the problem of computing lower and upper bounds for these lengths. Note that the VAS exhibiting a large (Ackermann size) but finite reachability set given in [12 does not directly provide an Ackermann lower-bound for these sizes since Presburger forward invariants can over-approximate reachability sets. Note that the existence of a primitive recursive upper bound of complexity for the reachability problem is still open since Zakaria Bouziane's paper [1] introducing such a bound was proved to be incorrect by Petr Jančar [6].

Acknowledgments: We thank Mikołaj Bojańczyk, Philippe Darondeau, Stéphane Demri, Alain Finkel, Pierre Ganty, Petr Jančar, Sławomir Lasota, Ranko Lazic, Sylvain Schmitz, Philippe Schnoebelen, Grégoire Sutre, and Marc Zeitoun for feedbacks and encouragement.

\section{References}

[1] Z. Bouziane. A primitive recursive algorithm for the general petri net reachability problem. In FOCS 1998, pages $130-136$, nov 1998.

[2] Javier Esparza and Marcus Nielsen. Decidability issues for petri nets - a survey. Bulletin of the European Association for Theoretical Computer Science, 52:245-262, 1994.

[3] Seymour Ginsburg and Edwin H. Spanier. Semigroups, Presburger formulas and languages. Pacific Journal of Mathematics, 16(2):285-296, 1966.

[4] John E. Hopcroft and Jean-Jacques Pansiot. On the reachability problem for 5-dimensional vector addition systems. Theoritical Computer Science, 8:135-159, 1979.

[5] Petr Jančar. Decidability of a temporal logic problem for petri nets. Theoretical Computer Science, 74(1):71 - 93, 1990.

[6] Petr Jančar. Bouziane's transformation of the petri net reachability problem and incorrectness of the related algorithm. Inf. Comput., 206:1259-1263, November 2008.

[7] S. Rao Kosaraju. Decidability of reachability in vector addition systems (preliminary version). In STOC 1982, pages 267-281. ACM, 1982.

[8] Jean Luc Lambert. A structure to decide reachability in petri nets. Theoretical Computer Science, 99(1):79-104, 1992.

[9] Jérôme Leroux. The general vector addition system reachability problem by Presburger inductive invariants. In $L I C S^{\prime} 09$, pages 4-13, 2009.

[10] Jérôme Leroux. Vector addition system reachability problem: a short self-contained proof. In POPL 2011, pages 307-316. ACM, 2011.

[11] Ernst W. Mayr. An algorithm for the general petri net reachability problem. In STOC 1981, pages 238-246. ACM, 1981.

[12] Ernst W. Mayr and Albert R. Meyer. The complexity of the finite containment problem for petri nets. J. ACM, 28(3):561-576, 1981.

[13] George S. Sacerdote and Richard L. Tenney. The decidability of the reachability problem for vector addition systems (preliminary version). In STOC 1977, pages 61-76. ACM, 1977.

[14] Alexander Schrijver. Theory of Linear and Integer Programming. John Wiley and Sons, New York, 1987. 